$\frac{12}{9} / 195950$

\title{
Opportunities and Challenges of High-Performance Computing in Chemistry
}

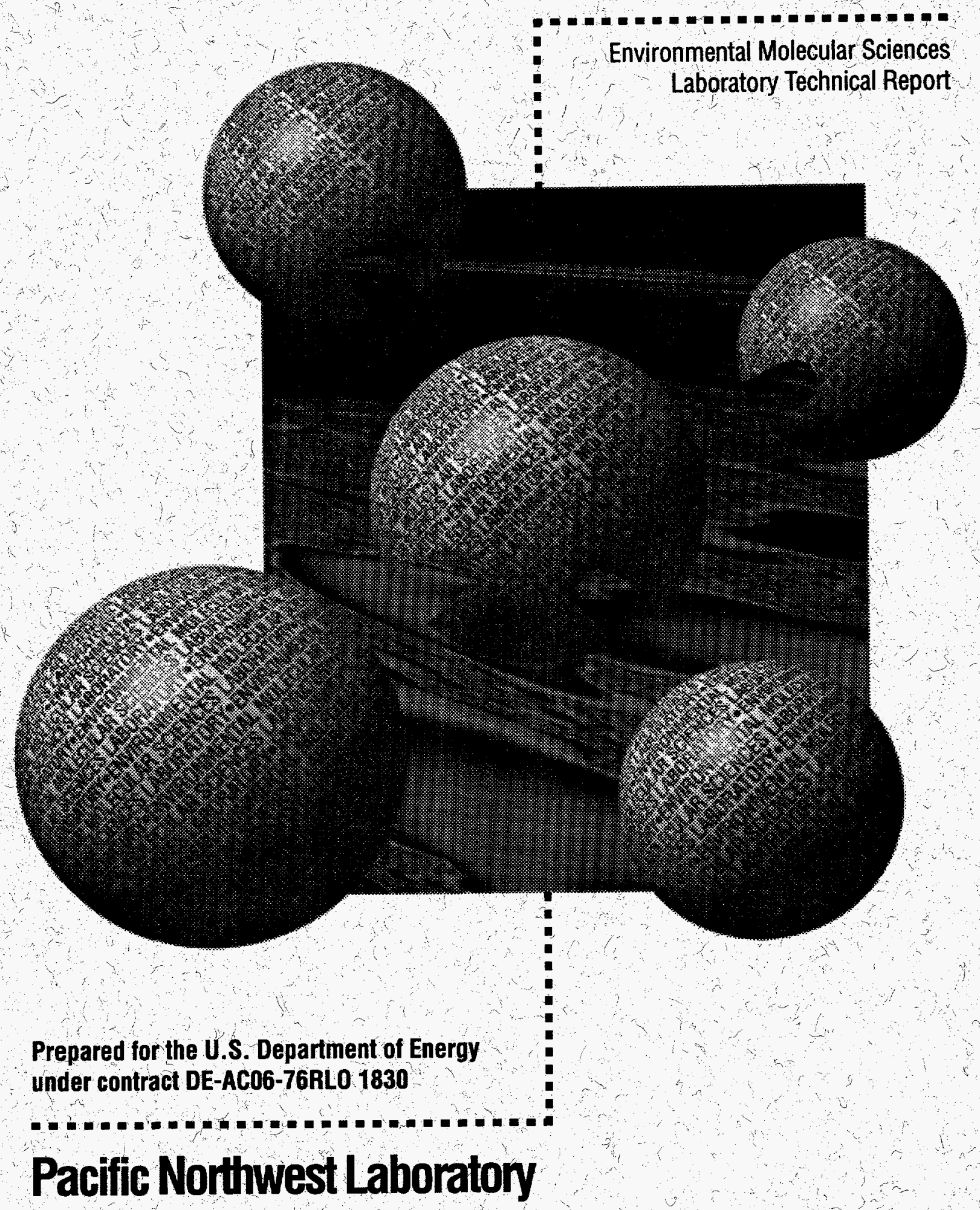

Operated by Battelle Memorial Institute for the U.S. Department of Energy 


\title{
DISCLAIMER
}

This report was prepared as an account of work sponsored by an agency of the United States Government. Neither the United States Government nor any agency thereof, nor Battelle Memorial Institute; nor any of their employees, makes any warranty, express or implied, or assumes any legal liability or responsibility for the accuracy, completeness, or usefulness of any information, apparatus, product, or process disclosed, or represents that its use would not infringe privately owned rights. Reference herein to any specific commercial product, process, or service by trade name, trademark, manufacturer, or otherwise does not necessarily constitute or imply its endorsement, recommendation, or favoring by the United States Government or any agency thereof, or Battelle Memorial Institute. The views and opinions of authors expressed herein do not necessarily state or reflect those of the United States Government or any agency thereof.

\author{
PACIFIC NORTHWEST LABORATORY \\ operated by \\ BATTELLE MEMORIAL INSTITUTE \\ for the \\ UNITED STATES DEPARTMENT OF ENERGY \\ under Contract DE-ACO6-76RLO 1830
}

Printed in the United States of America

Available to DOE and DOE contractors from the

Office of Scientific and Technical Information, P.O. Box 62, Oak Ridge, TN 37831;

prices available from (615) 576-8401.

Available to the public from the National Technical Information Service,

U.S. Department of Commerce, 5285 Port Royal Rd., Springfield, VA 22161 


\section{Opportunities and Challenges of High-Performance Computing in Chemistry}

Editors:

M.F. Guest, R.A. Kendall, J.A. Nichols,

T.H. Dunning, Jr., and M.S. Gordon

Authors:

J. Almlöf, R.A. Bair, B.R. Brooks, C.L. Brooks III, M. Colvin, D.A. Dixon, T.H. Dunning, Jr., M.F. Guest, R.J. Harrison, M. Kalos, A. Kuppermann, B. McCurdy, W.P. Reinhardt, A.P. Rendell, J. Saltz, K. Schulten, R. Stevens, P.R. Taylor, and A.F. Wagner

June 1995

Prepared for the National Science Foundation under grant CHE-9305615 and for the U.S. Department of Energy under contract DE-AC06-76RLO 1830

Pacific Northwest Laboratory Operated for the U.S. Department of Energy by Battelle Memorial Institute

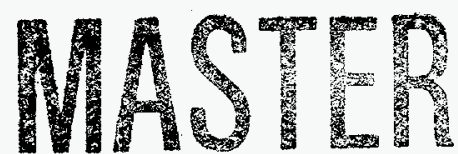




\section{DISCLAIMER}

Portions of this document may be illegible in electronic image products. Images are produced from the best available original document. 


\section{Summary}

The field of high-performance computing is developing at an extremely rapid pace. Massively parallel computers offering orders of magnitude increase in performance are under development by all the major computer vendors. Many sites now have production facilities that include massively parallel hardware. Molecular modeling methodologies (both quantum and classical) are also advancing at a brisk pace. The transition of molecular modeling software to a massively parallel computing environment offers many exciting opportunities, such as the accurate treatment of larger more complex molecular systems in routine fashion, and a viable, cost effective route to study physical, biological, and chemical "grand challenge" problems that are impractical on traditional vector supercomputers. This will have a broad effect on all areas of basic chemical science at academic research institutions and chemical, petroleum, and pharmaceutical industries in the United States, as well as chemical waste and environmental remediation processes. But, this transition also poses significant challenges: architectural issues (SIMD, MIMD, local memory, global memory, etc.) remain poorly understood and software development tools (compilers, debuggers, performance monitors, etc.) are not well developed. In addition, researchers that understand and wish to pursue the benefits offered by massively parallel computing are often hindered by the lack of expertise, hardware, and/or information at their site.

A conference and workshop organized to focus on these issues was held at the National Institute of Health, Bethesda, Maryland (February 1993). The specific aim of the conference was to provide a snapshot of current state-of-the-art and background material on the following:

1. current work in chemistry on parallel computers, with a special emphasis on massively parallel computers

2. the chemical problems which are likely to benefit substantially from the use of massively parallel computers

3. the impediments, programming and otherwise, preventing the migration of existing codes to, and the development of new codes for, massively parallel computers.

This report is the culmination of the organized workshop and the efforts of the participants; it discusses issues 1 and 3 at some length, and provides an update of activities that have taken place since the workshop. The main conclusion of the report is that a drastic acceleration in the present rate of progress is required for the chemistry community to be positioned to exploit fully the emerging class of Teraflop computers, even allowing for the significant work to date by the community in developing software for parallel architectures. 



\section{Recommendations}

The steps required to address this issue of increased rate of progress are encapsulated in eight specific recommendations, which are summarized below.

\section{Recommendation-1}

Recognizing that many of the scientifically driven needs of computational chemistry can only be met by future generations of massively parallel processors (MPP) computers, software and algorithm development should take both problem and resource scalability as a basic tenant.

\section{Recommendation-2}

Scalable parallel computational chemistry applications demand both advanced algorithm development and vendor-based hardware and software capabilities, targeted to the requirements of the computational chemistry community. A well-defined list of requirements must be tabulated, including those of portability, performance, enhanced development environments, scalable MPP subsystems (communication, disk I/O, memory capacity), compact expression of chemical algorithms, and available parallelism, including nonuniform memory access and multiple levels of granularity.

\section{Recommendation-3}

The development of new methods and algorithms should be encouraged and supported. A key aspect of this support should involve the integration of the computer science, computational science, and computational chemistry fields at the undergraduate and postgraduate levels.

\section{Recommendation-4}

Close interaction of computational chemistry application developers across chemistry disciplines and with computational scientists, computer scientists, and vendors should be strongly encouraged to foster coeducation of these varied communities.

\section{Recommendation-5}

Development and production use of MPP supercomputers are necessary to make full use of today's MPP systems, while preparing for the next generation of computing resources. Massively parallel processors supercomputer centers must be configured to meet the needs of computational chemistry applications and users.

\section{Recommendation-6}

MPP supercomputer centers should act as a repository for algorithms and expertise, and provide a focal point for communication between the computational communities, vendors, and application users. 


\section{Recommendation-7}

Technology transfer to industrial researchers must be streamlined to increase the productive use of MPP-based computational chemistry applications.

\section{Recommendation-8}

A suite of computational chemistry benchmarks (cf., the NAS kernels) needs to be developed to demonstrate requirements for comparison and development of software environments and hardware, as well as to provide performance measurements of MPP and conventional supercomputers. 


\section{Acknowledgments}

We would like to gratefully acknowledge the financial support of the National Science Foundation. This workshop in part was supported under the auspices of the Office of Scientific Computing, the Division of Chemical Sciences, Office of Basic Energy Sciences and the Environmental Management Office of the U.S. Department of Energy under contract DE-AC06-76RLO 1830 with Battelle Memorial Institute, which operates the Pacific Northwest Laboratory, a multiprogram national laboratory, and under contract W-31-109-ENG-38 at Argonne National Laboratory. We also would like to thank the National Institutes of Health for graciously hosting this workshop. We would also like to acknowledge the participation of many of the parallel computer vendors in providing a snapshot of their technology to the workshop participants. The editors would like to thank the authors and technical staff for their generous contributions of both time and ideas to this effort. 



\section{Acronyms}

$\begin{array}{ll}\text { CC } & \text { coupled cluster } \\ \text { CCSDT } & \text { coupled-cluster method, including all single, double, and triple excitations } \\ \text { CI } & \text { configuration interaction } \\ \text { CMM } & \text { Cell Multipole Method } \\ \text { CPHF } & \text { coupled Hartree-Fock } \\ \text { CSF } & \text { configuration state functions } \\ \text { DQMC } & \text { Diffusion Quantum Monte Carlo } \\ \text { FFT } & \text { Fast Fourier Transform } \\ \text { FME } & \text { Fast Multipole Expansion } \\ \text { GA } & \text { global array } \\ \text { GFMC } & \text { Green's Function Monte Carlo } \\ \text { GGA } & \text { general gradient approximation } \\ \text { HPCC } & \text { High Performance Computational Chemistry } \\ \text { HPF } & \text { High Performance FORTRAN } \\ \text { I/O } & \text { input/output } \\ \text { LDA } & \text { Local Density Approximation } \\ \text { LJ } & \text { Lennard Jones } \\ \text { LSD } & \text { Local Spin Density } \\ \text { MA } & \text { memory allocator } \\ \text { MBPT } & \text { Many-body perturbation theory } \\ \text { MD } & \text { Molecular Dynamics } \\ \text { MPI } & \text { message-passing interface } \\ \text { MPP } & \text { massively parallel processors } \\ \text { MRSDCI } & \text { Multireference single- and double-excitation configuration interaction } \\ \text { MTS } & \text { multiple time step } \\ \text { PARTI } & \text { (Parallel Automated Runtime Toolkit at ICASE) } \\ \text { QC } & \text { quantum chemistry } \\ \text { QMC } & \text { Quantum Monte Carlo } \\ \text { R\&D } & \text { research and development } \\ \text { SCF } & \text { self-consistent field } \\ \text { TCGMSG } & \text { Theoretical Chemistry Group MeSsaGe } \\ & \end{array}$





\section{Contents}

Summary $\ldots \ldots \ldots \ldots \ldots \ldots \ldots \ldots \ldots \ldots \ldots \ldots \ldots \ldots \ldots \ldots$ ii

Recommendations $\ldots \ldots \ldots \ldots \ldots \ldots \ldots \ldots \ldots \ldots \ldots \ldots$

Acknowledgments $\ldots \ldots \ldots \ldots \ldots \ldots \ldots \ldots \ldots \ldots \ldots \ldots \ldots \ldots \ldots$

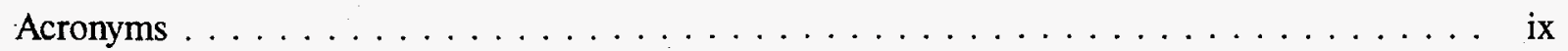

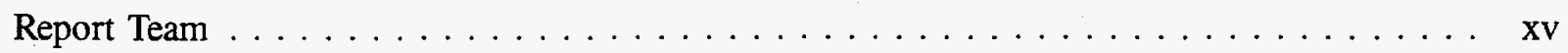

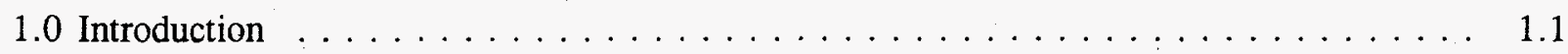

2.0 Computational Chemistry Software Development on High-Performance Computers $\ldots \ldots .2 .1$

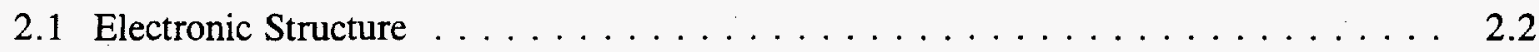

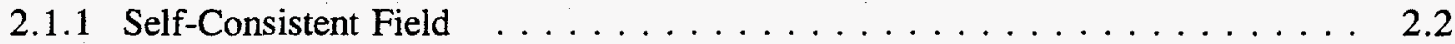

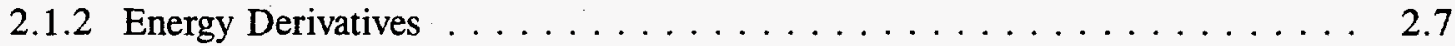

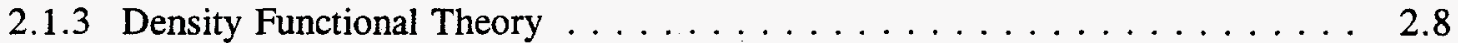

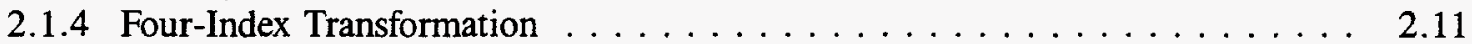

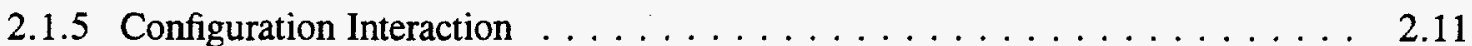

2.1 .6 Many-Body Methods . . . . . . . . . . . . . . . . . . . . . 2.13

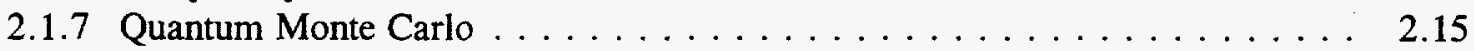

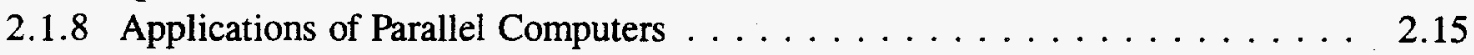

2.2 Quantum Reactive and Inelastic Dynamics . . . . . . . . . . . . . 2.16

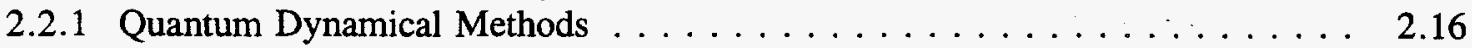

2.2 .2 Future Developments . . . . . . . . . . . . . . . 2.17

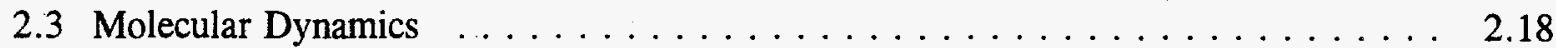

2.3.1 Computational Demands and Goals of Molecular Dynamics Simulations . . . . 2.18

2.3.2 Parallel Algorithms in Molecular Dynamics . . . . . . . . . . . . 2.20

2.3.3 Multiple-Timescale Molecular Dynamics Methods . . . . . . . . . . . 2.23

2.3.4 Parallel Simulation Packages . . . . . . . . . . . . . . . . . . 2.24

2.3.5 Benchmarking and Molecular Dynamics $\ldots \ldots \ldots \ldots \ldots \ldots \ldots$

3.0 Impediments to the Migration, Development, and Exploitation of Computational Chemistry Software on Massively Parallel Computers $\ldots \ldots \ldots \ldots \ldots \ldots \ldots \ldots \ldots$

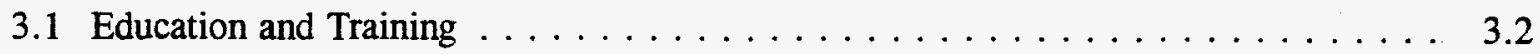

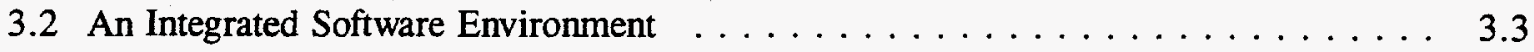

3.2.1 Compilers, Languages and Tools . . . . . . . . . . . . 3.3 


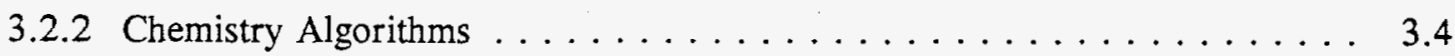

3.2 .3 Architectural Requirements . . . . . . . . . . . . . . 3.6

3.3 Integration of Communities $\ldots \ldots \ldots \ldots \ldots \ldots \ldots \ldots \ldots \ldots$

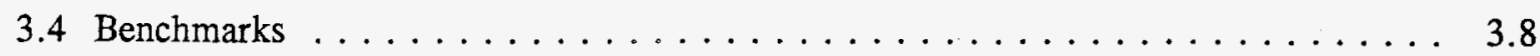

3.5 Configuration and Operation of Massively Parallel Processor Centers . . . . . . . 3.9

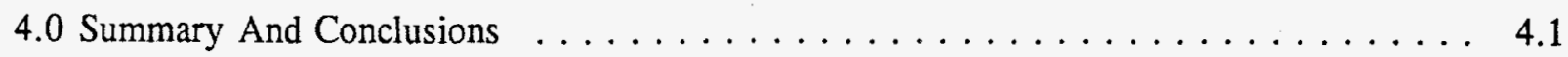

4.1 State-of-the-Art Software Developments . . . . . . . . . . . . . . . 4.1

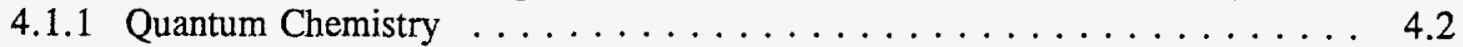

4.1 .2 Reactive Scattering . . . . . . . . . . . . . . . . . . 4.3

4.1 .3 Molecular Dynamics . . . . . . . . . . . . . . . . . 4.3

4.2 Impediments to the Migration, Development, and Exploitation

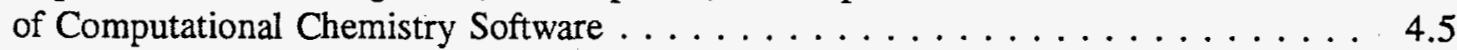

4.2 .1 Education and Training $\ldots \ldots \ldots \ldots \ldots \ldots \ldots \ldots \ldots . \ldots \ldots$

4.2.2 An Integrated Massively Parallel Processor and Distributed Computing

Environment for Chemistry . . . . . . . . . . . . . . . . 4.5

4.2.3 The Integration of Computational Communities . . . . . . . . . 4.5

4.2 .4 Computational Chemistry Benchmarks . . . . . . . . . . . . 4.5

4.2.5 Configuration and Operation of Massively Parallel Processor Centers . . . . . 4.5 


\section{Figures}

1.1 The Scope of Chemical, Physical, and Biological Research Areas that will Benefit from the Development of Computational Chemistry Application Software that is Scalable on Massively Parallel Supercomputers

1.2 Scales of Computational Complexity $\ldots \ldots \ldots \ldots \ldots \ldots \ldots \ldots$

2.1 The Scaling of the Components of the DFT Code (Wall Time Versus Number of Atomic Orbitals) on Various Zeolite Fragments Using 48 Nodes of the KSR-2 



\section{Report Team}

\section{Authors}

Jan Almlöf

Department of Chemistry

University of Minnesota

Minneapolis, MN 55455

e-mail:mf10101@uc.msc.umn.edu

Bernard R. Brooks

National Institute of Health

9000 Rockville Pike

Bethesda, MD 20892

e-mail: brbrooks@helix.nih.gov

Michael Colvin

Center of Computational Engineering

Sandia National Laboratory

Livermore, CA 94551

e-mail: mecolv@sandia.gov

Martyn F. Guest

Alistair P. Rendell

SERC Daresbury Laboratory

Warrington WA4 4AD

ENGLAND

e-mail: mf_guest@pnl.gov

apr@cxa.dl.ac.uk

Aron Kuppermann

Department of Chemistry

California Institute of Technology

1201 East California Boulevard

Pasadena, CA 91125

e-mail: aron@caltech.edu
Raymond A. Bair, Thomas H. Dunning, Jr.,

Robert J. Harrison

Environmental Molecular Sciences Laboratory

Pacific Northwest Laboratory

Richland, WA 99352

e-mail: ra_bair@pnl.gov

th_dunning@pnl.gov

rj_harrison@pnl.gov

Charles L. Brooks III

Department of Chemistry

Carnegie Mellon University

4400 Fifth Avenue

Pittsburgh, PA 15213

e-mail: brooks@scripps.edu

David A. Dixon

Du Pont Research and Development

Route 141 Murphy Road

Experimental Station

Wilmington, DE 19880-0328

e-mail: dixon@esvax.dnet.dupont.com

Malvin Kalos

Cornell Theory Center

Cornell University

Ithaca, NY 14853

e-mail: annak@tc.cornell.edu

Bill McCurdy

National Energy Research

Supercomputer Center

Lawrence Livermore National Laboratory

Livermore, CA 94551

e-mail: mccurdy@nersc.gov 
Authors (contd)

William P. Reinhardt

Joel Saltz

Department of Chemistry

Department of Computer Science

BG-10

University of Washington

University of Maryland

College Park, MD 20742

Seattle, WA 98195

e-mail: saltz@cs.umd.edu

e-mail: reinhard@uwchem.chem.washington.edu

Klaus Schulten

Department of Chemistry

University of Illinois

505 South Mathews Avenue

Urbana, IL 61801

e-mail: kschulte@lisboa.ks.uivc.edu

Rick Stevens

Math and Computer Science Division

Argonne National Laboratory

Argonne, IL 60439

e-mail: stevens@mcs.anl.gov

Peter Taylor

San Diego Supercomputer Center

Albert F. Wagner

Theoretical Chemistry Group

Chemistry Division

Argonne National Laboratory

PO Box 85608

San Diego, CA 92138

e-mail: taylor@cream.sdsc.edu

Argonne, IL 60439

e-mail: wagner@tcg.anl.gov

\section{Editors}

Martyn F. Guest, Rick A. Kendall, Jeffrey A. Nichols, and Thomas H. Dunning, Jr., Environmental and Molecular Sciences Laboratory

Pacific Northwest Jaboratory

Richland, WA 99352

e-mail: mf guest@pnl.gov

ra_kendall@pnl.gov

ja_nichols@pnl.gov

th_dunning@pnl.gov

Mark S. Gordon

Department of Chemistry

Iowa State University

Ames, lowa 50011

e-mail: mark@si.fi.ameslab.gov 
Technical and Staff Support

Rik L. Littlefield, John B. Nicholas, Mark A. Stave, Mark A. Thompson, and Deborah S. Baker

Environmental Molecular Science Laboratory

Pacific Northwest Laboratory

Richland, WA 99352

e-mail: rj_littlefield@pnl.gov

jb_nicholas@pnl.gov

msstave@cray.com

ma_thompson@pnl.gov

dsbaker@pnl.gov

Robert Martino

NIH/DCRT, CSL

Bldg. 12A, Rm. 2033

9000 Rockville Pike

Bethesda, MD 20892

e-mail: martino@alw.nih.gov

Organizing Committee

Chair: Thomas H. Dunning, Jr.

Raymond A. Bair

Robert J. Harrison

Rick A. Kendall

William P. Reinhardt e-mail: th_dunning@pnl.gov

ra_bair@pnl.gov

rj_harrison@pnl.gov

ra_kendall@pnl.gov

reinhard@uwchem.chem.washington.edu

\section{Advisory Committee}

Bernard R. Brooks

Martyn F. Guest

Robert Martino

Alfred F. Wagner e-mail: brbrooks@helix.nih.gov

mf guest@pnl.gov

martino@alw.nih.gov

wagner@tcg.anl.gov 


\subsection{Introduction}

The early 1990 s are proving to be a revolutionary time in computational science and, in particular, computational chemistry. Dramatic advances, which began in the $1980 \mathrm{~s}$, continue to gain momentum. In the 1980s, computational chemistry established itself as a full partner in chemical research, providing not only insights into the structure and dynamics of molecules, but also providing quantitative information which may be difficult or impossible to obtain in the laboratory. These advances are a result of the following:

- The development of new, more efficient algorithms for computational chemistry.

- Dramatic improvements in hardware cost/performance driven by advances in microprocessor technology.

- The availability of traditional supercomputer facilities critical for both development and production capabilities.

The former has not only decreased the computer resources required for a given calculation, but also has added significant new functionality. The latter has resulted in workstations with the computational capabilities of yesterday's supercomputers. The workstation revolution has also developed new visualization capabilities that are becoming a fundamental tool in the analysis of "computational chemistry experiments.

By the late 1990s, the above trends will be joined by the following:

- The delivery of scalable-parallel computers composed of thousands of high-speed processors connected by high-performance communications links.

- Software that will assist the computational scientists porting, tuning, and maintenance of their application base.

Scalable-parallel computing promises to revolutionize computational chemistry by harnessing the advances in microprocessor technology to solve the most challenging chemistry problems. It will allow us to extend the methods which have proven successful for small molecules to the treatment of larger, more complex molecular systems, and will also afford us the opportunity to develop entirely new innovative algorithms that are better suited for parallel computation, and possibly more suited for the large scale physical, biological, and chemical problems being tackled.

The development of scalable parallel computers and of molecular modeling software for these computers will greatly extend the role of computational chemistry in chemical research. With the computing power offered by these systems, it will be possible to plan and execute accurate simulations of complex chemical experiments completely ab initio. In addition, the chemical, petroleum, and pharmaceutical industries are finding that worldwide competition and environmental concerns demand an increasing reliance on predictive methods based on current theoretical methods. The increasing economic impact of computational chemistry applications provides a powerful driving force for the 
continuing development of associated techniques that will expand the predictive capabilities to physical, biological, and chemical systems that are orders of magnitude larger than the problems being solved on today's supercomputers.

All areas of chemical science that use computational chemistry as an effective research tool stand to benefit from the above developments. A snapshot of these areas and the breadth of the potential impact of scalable parallel computing is shown in Figure 1.1. This includes gas, liquid, and solid phase complex chemical systems that are a part of biological, chemical, chemical waste, industrial, and environmental processes that affect every citizen of the United States, as well as the global community. This revolutionary impact will come only in part from new computational hardware being delivered this decade. New software developed for these high performance massively parallel supercomputers will be required to deliver the promise of Teraflop computing before the turn of the century.

As an example, one specific grand challenge that must be addressed both experimentally and theoretically, using the developments mentioned above, is the waste remediation of chlorinated hydrocarbons, and in particular $\mathrm{CCl}_{4}$. Most industrialized countries did not stop using carbontetrachloride as a dry cleaning agent until the 1960s. Much of this waste was discharged directly to the environment. Carbontetrachloride was also used in many other chemical refining processes such as the plutonium refining process at Hanford. Simple models of chlorinated hydrocarbons in the environment will utilize accurate $a b$ initio through simulation methods that will incorporate molecular level interactions with transport models.

The development and implementation of these new methods as parallel supercomputer applications are a grand challenge in their own right. Computational chemistry algorithms span several orders of magnitude in time, length, and accuracy. A pictorial representation of this is given in Figure 1.2. As the size of a chemical, physical, or biological system increases, the demand on simulating this system is directly proportional to the required accuracy. Moreover, to replace or augment experimental science with simulations requires that the accuracy of a given simulation be known to reliable degrees of precision. It remains the case that current time and spatial scales in many areas of molecular simulation are often inappropriate for the target physical questions, a situation that is exacerbated by the lack of dedicated high performance computing resources.

The major aim of this report is to assess the current state-of-the-art in computational chemistry applications on parallel supercomputers, and to delineate the impediments to the development of these applications. Software developments are considered in Section 2.0, where we describe the current status of parallel algorithms and applications in the fields of electronic structure, quantum reactive and inelastic dynamics, and molecular dynamics. Notwithstanding the significant developments made by the community in developing software for parallel architectures, there can be little doubt that without a major acceleration in the present rate of progress, the community will not be positioned to fully exploit the emerging class of Teraflop computers. This theme is developed in Section 3.0, where we outline the impediments to both the development of parallel chemistry applications and the porting of existing codes. An analysis and summary of the current status of high-performance computational chemistry is presented in Section 4.0. Our recommendations (see pages $v$ and vi) for the steps that must be taken to ensure that the computational chemistry community is able to fully exploit and deliver on the promise of Teraflop computing are based both on this analysis, and on the panel's expertise in high performance computational chemistry. 


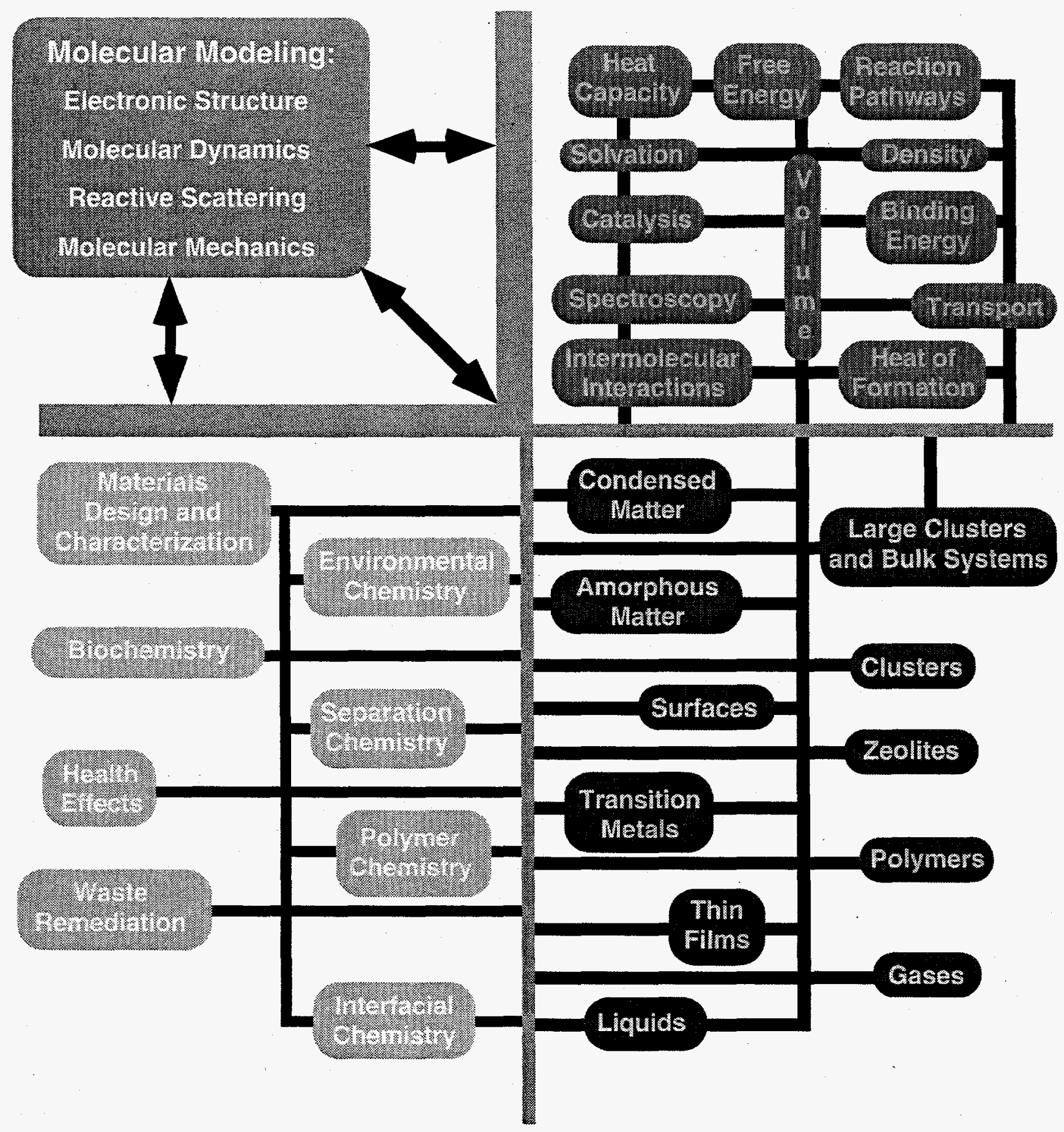

Figure 1.1. The Scope of Chemical, Physical, and Biological Research Areas that will Benefit from the Development of Computational Chemistry Application Software that is Scalable on Massively Parallel Supercomputers 


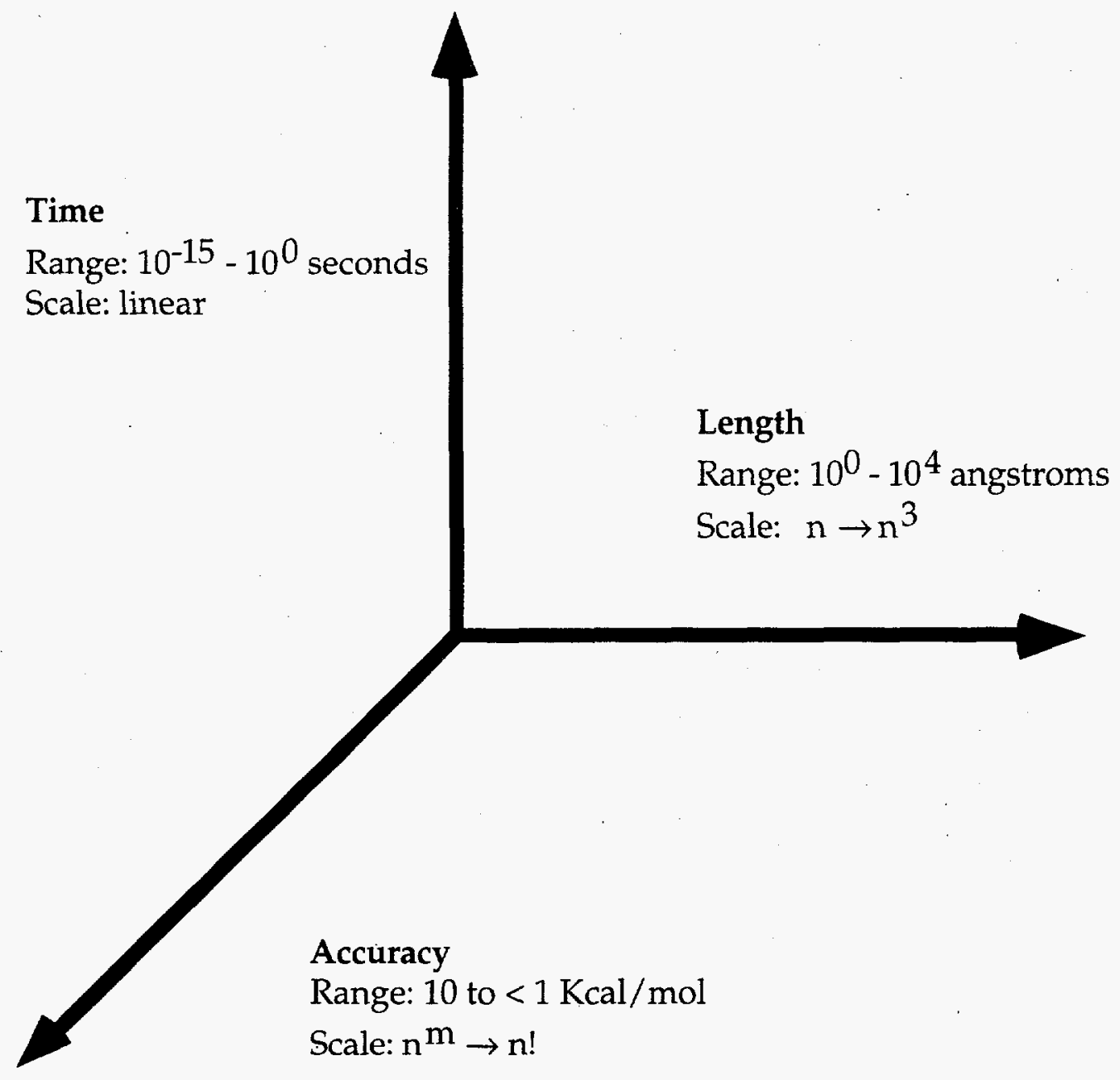

Figure 1.2. Scales of Computational Complexity

Several months after the Bethesda workshop, a CBO report, "Promoting High-Performance Computing and Communications", (ISBN 0-16-041828-3) focused on the role of the federal government in promoting new industries associated with the High Performance Computational Chemistry (HPCC) program. The report concentrated on the obstacles that might prevent the highperformance computing and data-communication industries from growing and using the technology being developed under the HPCC program. No recommendations were made, with the report primarily concerned with the commercialization of HPCC technology. Although little mention was paid to the specific issues in the area of molecular modeling, some of the more generic findings were common to those noted in the present report (e.g., the need for training and education). 


\subsection{Computational Chemistry Software Development on High-Performance Computers}

Parallel computing, as did previously vector computing, promises improvements in the peak performance, capacity, and cost effectiveness of computers. The ability of computational chemistry to realize this potential is, as with any other discipline, determined by effectiveness of the community in the development of algorithms and scalable application software capable of exploiting the underlying hardware. In this section we address specific issues related to the current state-of-the-art of computer codes and algorithms in quantum chemistry, reaction dynamics and molecular dynamics. We summarize a wide variety of attempts to exploit parallel computers and, from this, try to develop a sense of direction for these research areas. In particular, we hope to isolate some of the concepts and technologies being developed in successful applications, in the same manner that adoption of matrixoriented methods in quantum chemistry was identified as the key to high performance on vector supercomputers. Each subsection below is organized to assess the current status of parallel codes and associated algorithms, with an overview presented of work under way to migrate sequential implementations onto parallel architectures. Consideration is also given to those directions that will be required for continued progress in developing new codes on new machines.

Much of the current work remains based on replicated data algorithms with interprocessor communication handled by explicit message-passing. Clearly, as the molecular systems under study become larger, distributed data paradigms will be required to efficiently utilize current massively parallel processors (MPPs), and the Teraflop parallel supercomputers of the mid to late 1990s.

Appropriate data distributions have yet to be uncovered for many of the compute-intensive kernels in current chemistry codes, while our ability to solve much larger chemical, physical, and biological systems must surely necessitate a major effort in methods development. Computational chemistry application developers have a unique opportunity to make progress in both of these areas, by advancing new approaches which combine distributed data techniques along with the evolution of appropriate theoretical methods. These new applications should provide a progression for the development of truly scalable computational chemistry applications.

There are several recent reviews that consider this subject area. Wilson (1989) and Colvin et al. (1989) provide excellent, though dated, introductions to parallel ab initio methods, while Fincham (1987) and Plimpton (1994) have considered a variety of parallel algorithms and methods in molecular simulation. .Volumes 71, 72, and 84 of Theoretica Chimica Acta contain proceedings of conferences in which parallel ab initio chemistry featured strongly. Kendall et al. (1994) provide the most extensive general review of parallel computational chemistry and include much introductory material, along with a survey of published abstracts and articles. A more focused review of parallel ab initio methods in quantum chemistry is due to Harrison and Shepard (1994). Our summary of state-of-the-art software developments draws heavily upon material from these two reviews.

Finally, let us reconsider our objectives of increased peak performance and cost effectiveness. The first objective refers to MPPs, or parallel supercomputers, which are, by definition, expensive. If the goal is reduced execution time without regard to price, then processor utilization is the appropriate metric to determine efficient usage. However, if the goal is to perform larger calculations, other 
machine resources such as memory and disk bandwidth become important. The second objective of increased cost effectiveness also emphasizes efficient usage of all machine resources, and we note that processors are a small fraction (usually less than 10\%) of the cost of a typical workstation farm or parallel computer purchased for chemistry production and development.

\subsection{Electronic Structure}

At the heart of theoretical chemistry lies the calculation of the electronic wave function. Essentially, all of the chemical and physical properties of molecules, including the forces between two molecules or between a molecule and a solid surface, are determined by the quantum mechanical behavior of the electrons which form the bonds between atoms and molecules. The electronic wave function is obtained by solving the Schrödinger equation, either by using standard algebraic techniques, or by little-explored numerical approaches, which appear at first sight to be well-suited for parallel computations. In fact, there are many theoretical models and algorithms that have not been thoroughly explored because of the perceived cost of their sequential or vector implementations and limited computational resources.

The Hartree-Fock method approximates the electronic wave function as a simple (antisymmetric) product of orbitals, one for each electron in the molecule. The scaling of Hartree-Fock calculations is determined by the calculation of integrals over the basis functions used to expand these orbitals and by the subsequent assembly of the Fock matrix. These two processes both scale as $n^{4}$, where $n$ is the number of basis functions (proportional to the number of electrons). Once a molecule becomes sufficiently large, many of the component atoms are so distant from one another that the associated integrals can often be neglected (not computed) or approximated by multipole expansions. In this limit, the scaling reduces to $\mathrm{O}\left(\mathrm{n}^{2}\right)$. It is currently feasible to do accurate Hartree-Fock calculations on systems of 20 to 50 atoms.

Although the Hartree-Fock wave function describes many of the qualitative features of the electronic structure of molecules, the accurate prediction of many molecular properties (e.g., bond energies and reaction barriers) requires that electron correlation be explicitly taken into account. Configuration interaction ( $\mathrm{CI}$, based on both single and multireference wave functions), many-body perturbation theory, and coupled cluster methods are widely used to treat correlation. These methods scale far more dramatically with basis set size than the Hartree-Fock method. For example, second order perturbation theory (MP2) scales as $\mathrm{n}^{5}$, multireference singles and doubles $\mathrm{CI}$ as $\mathbf{M n}^{6}$ (where $\mathrm{M}$ is the number of reference configurations), while the coupled-cluster method, including all single, double, and triple excitations (CCSDT), scales as $\mathbf{n}^{8}$.

\subsubsection{Self-Consistent Field}

Parallel self-consistent field (SCF) algorithms dominate work performed to date in exploiting parallel machines. The input/output (I/O) problem of storing and repetitively processing the twoelectron integrals in conventional SCF is avoided in direct schemes by (re-)computing the integrals as required. This is particularly pertinent to current MPP architectures which generally provide very high computation rates and very poor I/O rates. Since each of the two-electron integrals may, of course, be computed independently, integral evaluation has very high parallelism, although for efficiency, the 
integrals must be computed in shell blocks (a shell block is a group of functions on the same center with common radial factor). This blocking, and possible contraction into symmetry adapted functions, causes the construction effort for various integral blocks to differ by three or more orders of magnitude. In addition, it is necessary to prescreen to avoid computation of integrals that are negligible. The variation in complexity of the integrals and the associated lack of regularity in the computation almost certainly preclude an efficient SIMD implementation because of poor loadbalancing.

The contraction of the integrals with the density matrix to form the Fock matrix requires that the integrals and required density and Fock matrix elements are brought together. The vast majority of parallel SCF programs, even on shared-memory computers, replicate the Fock and density matrices within each parallel process. The elements of the density matrix are then locally accessible, and a partial Fock matrix is formed locally. The partial Fock matrices are summed to form the total matrix only when all integrals have been computed. For this approach, there are several powerful arguments, as follows:

- Only a handful of lines of a sequential SCF program need be modified.

- Very simple parallel programming tools, in particular message-passing, are adequate for the task.

- High efficiency may be obtained for the Fock-matrix construction since there is no communication apart from the broadcast of the density and the global summation of the Fock matrix each iteration.

- The parallelism is very coarse grained and so can utilize machines with high overheads for process creation or communication (e.g., a global network of supercomputers).

- Full advantage may be taken of the permutational symmetry in the integrals and no additional computation need be introduced.

However, there are also profound drawbacks to this replicated-data strategy which strike right at the heart of the original motivations for using parallel computers. First, the maximum problem size is limited by the memory of each processor, rather than the aggregate memory of the machine. Second, the cost-efficiency of parallel computing is greatly compromised. Neglecting sparsity and assuming that perfect parallelism is achieved, the number of processors, $P$, required to construct the Fock matrix for $\mathrm{N}_{\text {basis }}$ functions in time , $\mathrm{T}, \mathrm{P}(\mathrm{T})=\mathrm{O}\left(\mathrm{N}_{\text {basis }}^{4} / \mathrm{T}\right)$. Thus, the memory (M, a large component of the cost of any high-performance computer) required by the replicated data approach, $M(T)=O\left(P * N_{\text {basis }}{ }^{2}\right)$ $=\mathrm{O}\left(\mathrm{N}_{\text {basis }}{ }^{6} / \mathrm{T}\right)$, in contrast to the optimal $\mathrm{O}\left(\mathrm{N}_{\text {basis }}{ }^{2}\right)$. Algorithms, which distribute data between processes, are essential to realize the full benefits of parallel computing. Various schemes have been adopted to address these concerns.

We consider, in more detail, variations on parallel SCF algorithms: first, replicated-data and then, distributed-data approaches. We then conclude this section by examining other issues pertinent to efficient computation of SCF wavefunctions and molecular properties. 


\subsubsection{Replicated-Data Algorithms}

The initial ground-breaking work undertaken by Clementi and co-workers at IBM in the parallelization of SCF on the LCAP systems, and also by Ostlund and Colvin et al. on the Intel iPSC, has provided the foundation on which future efforts have been based. Ostlund and Colvin were among the first to emphasize the design of scalable algorithms, anticipating the availability of highly-parallel computers with many tens or hundreds of processors.

Some of the early message-passing environments emphasized portability and, to a lesser extent, efficiency, but not robustness. These issues led to the development of a portable message-passing toolkit, Theoretical Chemistry Group MeSsaGe passing library (TCGMSG), a small library that provides simple message passing, global operations, and a tool for dynamic load-balancing (Harrison 1991). The simplicity of the model makes it easy to use and very portable, while the provision of global operations and dynamic load-balancing make replicated-data algorithms straightforward to implement. Several other packages (e.g., network Linda, Express, P4, PVM) provide more functionality than TCGMSG and have been compared for use in performing SCF calculations on a network of workstations. The recently proposed standard message-passing interface (MPI) provides many powerful features and eliminates many of the unsafe and ill-defined features of other packages.

In the late 1980s, shared-memory parallel vector supercomputers (such as the CRAY X-MP and CRAY2) and minisupercomputers (such as Alliant and Convex) were becoming widely available. The parallel computing environments on these machines evolved from explicit shared-memory parallel programming methods to automatically parallelizing FORTRAN and C compilers. Even now the compilers are still unable to automatically analyze programs as complex as a direct-SCF code. Therefore, even using a parallelizing compiler, the programmer must shoulder all responsibility for correct parallelization of the algorithm. Such compilation environments do provide sharing of data between different processes or threads of execution, making it possible, in principle, to avoid the replication of data within processes. A good example of the implementation of SCF codes on parallel vector machines is provided by Luthi et al. (1992), who employed the Cray microtasking and autotasking compiler directives to parallelize the vectorized direct-SCF program, DISCO, on a CRAY Y-MP/8-128. Impressive performance was obtained with speedups of 7.7 to 7.9 and computation rates in excess of 1.5 GFLOPS on the CRAY Y-MP/8-128.

Various performance and programming issues, however, still make replicated-data models attractive on shared-memory computers. First, the message-passing paradigm is trivially portable between both shared- and distributed-memory machines and requires only a small modification to the sequential code. Second, some parallelizing compilers still do not provide facilities for labeling common blocks as not shared or thread local. Thus, substantial restructuring can be required for common blocks that are updated within a parallel loop. Third, the mutual exclusion required around the accumulation of integrals into the shared Fock matrix is a bottleneck unless done at a sufficiently coarse grain which again increases code complexity. Finally, the memory hierarchy of many sharedmemory parallel computers makes the updating of shared data at a fine grain inefficient. Thus, the efficient use of shared-memory models raises many of the same design issues found in distributedmemory models. 
On distributed memory computers, the simplicity of the replicated-data approach and its robust performance on few processor machines has lead to its widespread use in research and production codes. Brode et al. (1993) described a parallel version of TURBOMOLE, distinguished by its application to improve turnaround time in the solution of industrially important problems, and by a thorough analysis of load-balancing issues in the context of high-symmetry semidirect calculations.

The direct-SCF program, DISCO, and the related SUPERMOLECULE have been used for many parallel implementations, notably by Luthi, Feyereisen, and Vogel. Luthi and Almlof (1993) used an asynchronous remote procedure call mechanism and a modification of the previous shared-memory version loop structure to distribute an SCF computation across multiple Cray computers, including simultaneous use of machines in Minnesota and Switzerland. On a local network of five dedicated CRAY Y-MP computers with a total of 20 processors, a speedup of 16.6 , and a performance of 3.3 GFLOPS was obtained. Slow wide area networks and load imbalance limited performance. A more detailed performance model is developed for this program on a network of IBM workstations by Vogel (1993). Kendall, Feyereisen and co-workers (1993) use a master process to hand out tasks comprising multiple shell blocks of integrals. The Fock-matrix construction realized a best speedup of 300 on 512 processors using the Intel Touchstone Delta, the degradation being ascribed to global communications, and the single master process being unable to respond sufficiently fast to 511 slaves.

The parallel $a b$ initio chemistry packages that have arguably had the most widespread use are the independent versions of the GAMESS package: GAMESS-UK (Guest 1993) and GAMESS-USA (Schmidt 1993). Both feature parallelized energy and gradients for a variety of SCF wavefunctions using both conventional and direct algorithms, with dynamic load balancing. A single point 389 basis function SCF gradient calculation, using GAMESS-USA, obtained an overall speedup of about 100 on 512 nodes of the Intel Touchstone Delta.

\subsubsection{Distributed-Data Algorithms}

The replicated-data strategy described above is inherently nonscalable and does not address the issues of providing qualitatively improved capability and cost-effectiveness through the use of massively parallel processors. Fully distributed algorithms are required to meet these goals.

The systolic loop algorithm used by Ostlund and Colvin (1993) was, until recently, the most successful distributed-data method using message passing. The Fock and density matrices are divided into matching blocks and statically assigned to processors. A second copy of the Fock and density matrices are similarly partitioned but circulate in a systolic loop of processors. At every step, each processor forms all interactions connecting the current density and Fock matrix elements and then passes data to the left and receives new data from the right. Although only the unique pairings of Fock and density matrix elements are produced, the unique set of integrals have to be evaluated three times. Since the entire Fock and density matrices must flow through each processor, the best possible execution time is $\mathrm{O}\left(\mathrm{N}_{\text {besis }}{ }^{2}\right)$. Load balancing problems forced Colvin to adopt asynchronous communications and double buffering since the systolic nature of the algorithm precluded dynamic load balancing. Overall efficiencies of approximately $50 \%$ were obtained on 256 processors of an nCUBE-2 for a 164 basis function calculation on the cytosine dimer. Relative to the time taken to compute the unique list of integrals sequentially, this efficiency would be further reduced by a factor of three. 
Despite these inefficiencies, Colvin convincingly demonstrated the cost-effectiveness and improved scalability of their algorithm in comparisons with the performance of GAUSSIAN-92 on a CRAYYMP.

The most efficient explicit message-passing algorithm seems to be that due to Furlani and King (1994). The basis set is partitioned into blocks which do not break shell boundaries and contain at least $\mathrm{N}_{\mathrm{b}}$ functions. This blocking of the basis is then used to block the matrices, the blocks being assigned to individual processors. The computation of the unique two-electron integrals is divided into tasks corresponding to a quartet of blocks of basis functions. Approximately $40 \%$ of these tasks are statically assigned to processors with care to minimize communication, and the remainder are dynamically assigned with a variable granularity upon request to a control or master process. Each task requires access to up to six blocks of the Fock and density matrices. Reuse of density matrix blocks and caching of writes to Fock matrix blocks reduces the average number of blocks of each matrix that must be fetched to three. Requests for density matrix elements are again issued in advance using asynchronous communication. Excellent performance is reported using dynamic load balancing on an Intel iPSC-2 with a speed increase of 3.85 reported for a 204 basis function calculation in scaling from 4 to 16 processors (an Amdahl's law model provides a 16 node speedup as 15.25).

The complexity of these message-passing algorithms, and the simplicity of SCF in comparison with other $a b$ initio algorithms, clearly points to the need for more appropriate programming models. Harrison and co-workers (1994) have been investigating the use of distributed data structures that may be independently accessed in the same fashion as "real" shared memory, even on distributed memory machines. A significant performance enhancement also arises from increased asynchrony of execution of processes. Message passing forces processes to cooperate (e.g., by responding to requests for a particular datum). Inevitably, this involves waiting for a collaborating process to reach the same point in the algorithm, which is only partially reduced by the use of complex buffering and asynchronous communication strategies. With a one-sided communication mechanism, each process can access data without arranged cooperation of another process, and all processes can operate independently. This eliminates unnecessary synchronization and naturally leads to interleaving of computation and communication. The latest program from Harrison is built using tools that support just one- and twodimensional distributed arrays. The tools are simple and portable, being implemented on the Touchstone Delta, shared-memory UNIX processors, and workstation networks. Both data-parallel and task-parallel operations are efficiently supported and some simple linear algebra operations are provided. All computational steps of the SCF with complexity greater than $O\left(N_{\text {atom }}\right)$ have been parallelized. The four nested loops over the unique integrals are stripmined into blocks, similar in spirit to Furlani and King, where a block comprises all basis functions on an atom. The geometrical decomposition permits sparsity to be used to screen all references to global data. A task comprises computation of all unique integrals for up to five quartets of atom labels and their accumulation into the Fock matrix. Assignment of multiple atom quartets to a task improves caching of reads of the density matrix and accumulation into the Fock matrix, though too large a task size degrades load balancing. All tasks are dynamically assigned. This distributed array toolkit provides an operation to accumulate into a shared array with the necessary mutual exclusion.

A simple performance model predicts a constant efficiency for the Fock-matrix construction of about $99 \%$ for up to $\mathrm{O}\left(\mathrm{N}_{\text {atom }}{ }^{2}\right)$ processors (for extended molecular systems) at which point load balancing will degrade performance. Overall SCF efficiency is limited by the diagonalization which, although running in parallel, achieves only a modest speedup for these systems. The largest calculation 
(731 functions) using 512 processors of the Intel Delta obtained a speedup for the Fock-matrix construction of 496 (97\% efficient) while the entire SCF calculation had a speedup of only 438 (86\% efficient).

\subsubsection{The Diagonalization Bottleneck}

A recurring theme in the discussion above has been that the parallel scalability of SCF is greatly diminished by sequential bottlenecks and, in particular, the diagonalization step. Parallel diagonalization has been discussed by many workers, with various approaches reported that enable a small number of processors to be successfully exploited. These include performing the diagonalization of independent symmetry blocks in parallel and, for periodic codes (e.g., CRYSTAL), performing the diagonalization for each point in reciprocal space in parallel. Such approaches do not, however, address fully-distributed methods or low-symmetry problems. The situation is even less favorable when considering the new algorithms being adopted for fast construction of the Fock matrix, including multipole approximations, approximate factorizations of the two-electron integrals, and pseudo-spectral methods.

Littlefield (1993) provides the most objective discussion and compares the performance of several algorithms and implementations on the Intel Touchstone Delta. For large (dimension greater than 1,000 ) matrices, good speedup may be obtained, but communication overhead rapidly diminishes the performance for smaller matrices. In addition, the best algorithm varies with the number of processors and matrix size. Iterative methods, especially restricted to just the required occupied orbitals, also realize some advantage.

Rather than adopt an ad hoc approach, along with the additional code required for reliable convergence using DIIS, Harrison and co-workers are exploring the use of second-order convergent methods, as originally used for SCF by Bacskay (1981). The diagonalization is replaced with a matrix inversion, matrix multiplications, and additional constructions of Fock-like matrices. The sequential operation count of a matrix inversion is approximately 2 to 3 times less than that of a diagonalization, and it also has superior parallel efficiency. The second-order approach provides reliable convergence and also the necessary functionality to compute response properties by solution of the coupled HartreeFock (CPHF) equations. Wong and Harrison (1994) have shown that careful analysis of required convergence tolerances and integral selection thresholds results in less than twice as many integrals being computed by the second-order scheme with poor initial orbitals, with a significant advantage being gained if good starting orbitals are available. Tilson and Shepard are exploring simultaneous construction of multiple Fock matrices. Furthermore, faster integral evaluation, improved screening, massive parallelism, and fast Fock-matrix construction methods all greatly favor the second-order approach.

\subsubsection{Energy Derivatives}

The most expensive contribution to the derivative of the energy. with respect to a nuclear coordinate is due to the derivatives of the two-electron integrals. Computation of this sum is clearly massively parallel and one advantage of the replicated-data approach is that very little must be changed to 
parallelize computation of the derivatives. Thus, many authors have reported impressive efficiencies for the evaluation of derivatives with respect to nuclear coordinates. The gradients may be used for geometry optimizations, numerical Hessian-evaluation, and ab initio molecular dynamics.

Windus et al. (1993) describe the first parallel algorithm for analytic evaluation of the second derivatives of the SCF energy with respect to nuclear coordinates, in which the sequential four-index transformation is overlapped with parallel computation of the derivative integrals. Subsequently, the CPHF equations are solved sequentially. Dynamic load balancing and replication of data are used and a useful twofold speedup is obtained on three workstations. Most recently, Dupuis (1994) has a full implementation of parallel analytic second-derivatives within HONDO for the IBM SP-1. All steps, including derivative integral evaluation, four-index transformation, and CPHF calculation in the MO basis, execute in parallel with no redundant operations. The fast communications of the SP-1 switch are required for an efficient four-index transformation (see below).

Solution of the CPHF or response equations can be formulated as an iterative process requiring construction of Fock-like matrices. Thus, properties such as polarizabilities, which may be formulated as second and higher derivatives of the SCF energy with respect to external fields, are readily computed. Dupuis et al. (1987) analytically computed up to third derivatives of the energy with respect to an external electric field reusing the AO-based Fock-matrix construction, from disk-resident integrals, of their parallel SCF program. Feyereisen et al. (1993) extended their replicated-data message-passing parallel version of the direct-SCF program, DISCO, to compute frequency-dependent polarizabilities, which was applied by Nichols and Simons (1993).

\subsubsection{Density Functional Theory}

There are many density functional theory software packages available in the chemistry community; DGauss, deMon, DeFT, DMOL, GAUSSIAN-92, CADPAC, MOLPRO, Numol, Amol (ADF), QChem, Quest, TX93, CRYSTAL, and NWChem are some of those which come to mind. They are implementations of the Hohenberg-Kohn-Sham formalism (Hohenberg and Kohn 1964; Kohn and Sham 1965) of DFT. DFT is able to give results similar to those from correlated ab initio methods at reduced computational expense; it assumes an approximate expression for the energy in terms of functionals of the electronic charge density. An active area of research involves the development of more accurate energy functionals (successively better approximations to the Hamiltonian, in particular the exchange-correlation functional). This contrasts with traditional ab initio molecular orbital methods which assume an exact Hamiltonian and successively better approximates the wavefunction.

There is a raft of heavily utilized and highly successful plane wave codes which were not mentioned; for example, codes based on Car and Parrinello's implementation (e.g., AIMD) or the implementation of Teter, Payne, and Allen (e.g., CORNING). These codes have been of particular importance to the physics community, for example, in the study of metal systems, but have been less successful in treating many of the molecular systems of interest to the chemistry community. The latter shortcoming led to the emergence of the Gaussian-based and purely numerical implementations.

In the Gaussian basis DFT method the Hamiltonian is broken down into the same basic oneelectron and two-electron components as traditional methods, with the two-electron component further reduced to a Coulomb term and an exchange-correlation term. The treatment of the former can be 
accomplished in a fashion identical to that used in traditional SCF methods, from a fitted expression like that found in RI-SCF, or from the commonly used Dunlap fit. DFT is really distinguished from other traditional methods, however, by the treatment of the exchange-correlation term. This term is typically integrated numerically on a grid, or fit to a Gaussian basis and subsequently integrated analytically.

In almost all cases, the codes mentioned above have incorporated the exchange-correlation functionals (Dirac's exchange functional [Dirac 1930] and Vosko, Wilk, Nusair's correlation functional [Vosko, Wilk, and Nusair 1980]) required to obtain the Local Density Approximation (LDA) and Local Spin Density Approximation (LSD) for open shell systems. In addition, some form of the general gradient approximation (GGA) for the exchange-correlation contribution has been implemented (e.g., the popular Becke exchange functional [Becke 1988] and the Lee, Yang, Parr correlation functional [Lee, Yang, and Parr 1988]).

Most of these packages have incorporated several techniques to improve convergence in the iterative SCF procedure, such as damping, level shifting, and DIIS. Geometry optimization has become routine, with energy gradients available in almost all cases, although these can differ significantly based on types of fitting implementation and philosophy surrounding proper differentiation of energy components (e.g., quadrature weights). There have been mixed reviews recently regarding the use of a fitting basis for the exchange-correlation potential, since it leads to a procedure which is no longer variational and complicates the energy derivatives. In a few cases, analytical second derivatives are available (e.g., GAUSSIAN92, DGauss, etc.).

Many of these software packages share common attributes. The DFT implementations of GAUSSIAN-92, CADPAC, MOLPRO, TX93, and CRYSTAL originate from previously existing SCF software. They build Coulomb contributions accordingly (an analytical $\mathrm{O}\left[\mathrm{N}^{4}\right]$ method as previously discussed), and integrate the exchange-correlation contribution using standard grid technology. DGauss, deMon, DeFT, and NWChem are $\mathrm{O}\left(\mathrm{N}^{3}\right)$ methods relying on a fit of both the charge density and exchange-correlation functional in terms of auxiliary Gaussian basis sets. DMOL, Numol, and Amol (ADF) are numerical implementations of the KS equations; DMOL and ADF using the LCAO approximation with numerical basis functions, whereas Numol does not (ADF can, in addition, use Gaussian and Slater type functions). Quest is a periodic DFT implementation developed over a period of years at Sandia National Laboratory (Feibelman, Sears, and Schultz). The immediate impressive scaling of $\mathrm{O}\left(\mathrm{N}^{1.4-1 .}\right)$ is somewhat tempered by limited functionality. As the code currently stands, its capabilities include molecular and periodic solutions for LDA and forces. Its scaling is a result of partitioning the density into two components: an atomic component, which is calculated once only $\left(\mathrm{O}\left[\mathrm{N}^{3}\right]\right)$, and the remainder, which is updated each iteration and evaluated numerically on an even spaced 3-D grid (with spacings of $0.25-0.5 \mathrm{bohr}$, depending on the system).

$$
\rho=\sum_{a} \rho^{a}+\sum_{\text {grid }} \rho^{\text {rem }}, F^{n}=F^{a}+F^{\text {rem }}
$$

The atomic densities are considered the rapidly varying component and the remainder slowly varying and therefore easily fit (with a coarse grid). The rapidly varying component is constructed from pseudopotentials. This is to ensure that none of that atomic component creeps into the slowly varying piece, but this is an unfortunate limitation when one desires properties where an all-electron description is needed. The periodic implementation is accomplished by making sure all objects are 
charge neutral and summing the rapidly varying contributions until convergence is met. The slowly varying component is Fourier transformed to $\mathrm{k}$ space (taking advantage of the regular grid).

Of these, DGauss, NWChem, DMOL, Quest, and (potentially) QChem have parallel capabilities and only NWChem and Quest have been implemented in a manner to exploit MPP technology with maximum efficiencies. NWChem heavily utilizes the tools developed in the HPCC group; for example, the dynamic memory allocator (MA), the TCGMSG, and the global array (GA) tools. All data greater then $\mathrm{O}(\mathrm{N})$ is stored in global arrays. The four time consuming steps in NWChem's DFT are fit of the charge density, calculation of the Coulomb potential, evaluation of the exchangecorrelation potential, and diagonalization of the Fock matrix. Of course, given a DFT energy expression, differentiating it with respect to variational parameters leads to the self-consistent KohnSham equations, which are remarkably similar to the Hartree-Fock equations of molecular orbital theory (as described above), and hence parallelization of these equations proceeds analogously. The same strip-mining over atomic centers, etc., as exploited in SCF can be exploited here. Figure 2.1 shows the scaling of each of the components mentioned above as well as the overall scaling of the DFT code on zeolite fragments using 48 nodes of the Kendall Square KSR-2.

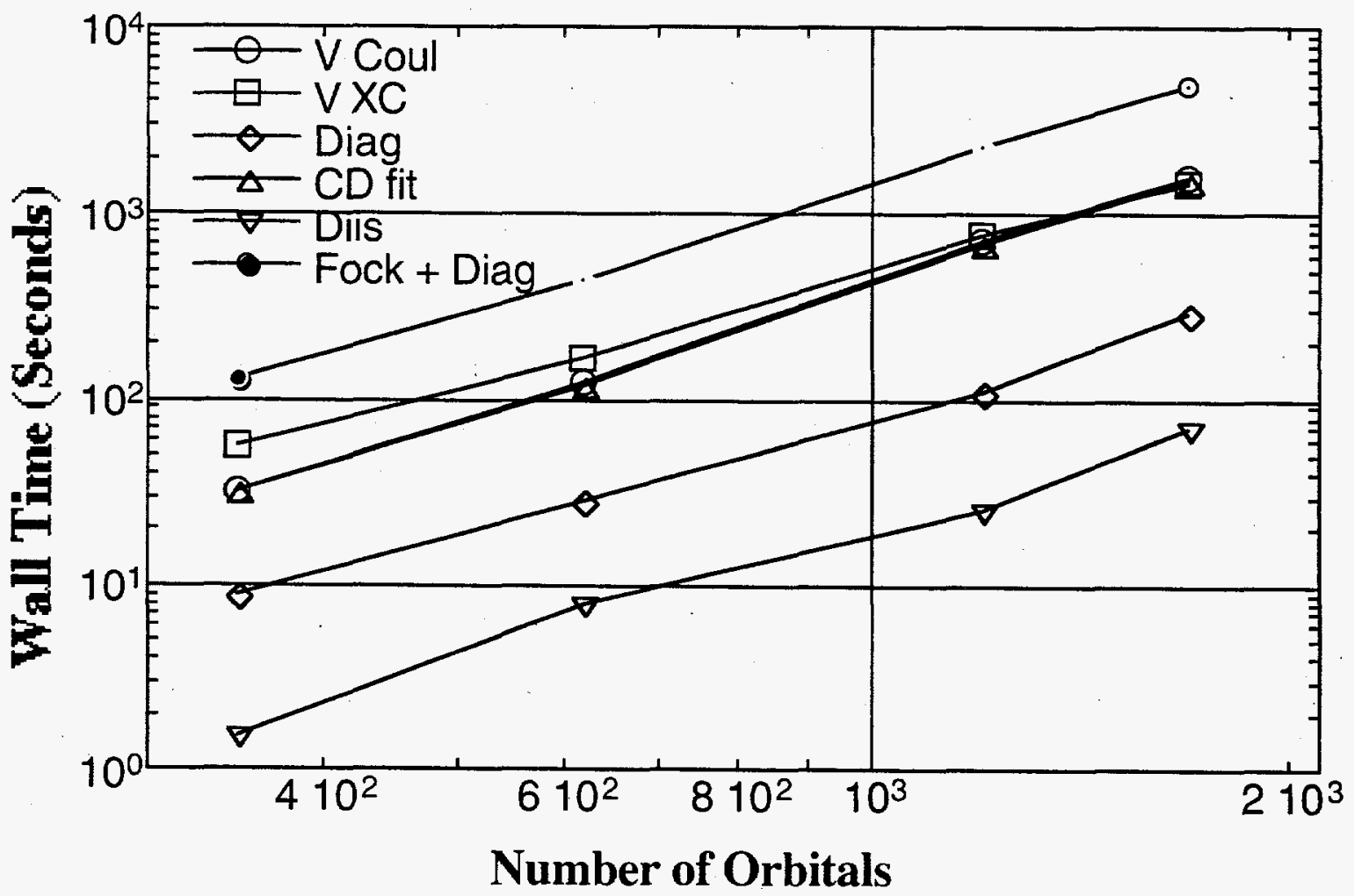

Figure 2.1. The Scaling of the Components of the DFT Code (Wall Time Versus Number of Atomic Orbitals) on Various Zeolite Fragments Using 48 Nodes of the KSR-2. In a fit to the function $t=\mathrm{An}^{\mathrm{B}}$ for each of the components the Value B obtained was $2.47,2.50,2.11,2.15,2.32,2.33$, for CD fit, $\mathrm{V}_{\text {coul }}, \mathrm{V}_{\mathrm{XC}}$, Diag, DIIS, and Fock+Diag, respectively. 


\subsubsection{Four-Index Transformation}

Most post-Hartree-Fock electronic structure methods require the one- and two-electron integrals in the MO basis which are generated by transformation of the AO integrals, formulated as successive oneindex transformations with storage of the partially-transformed arrays, the cost of the full two-electron integral transformation is $\mathrm{O}\left(\mathrm{N}_{\text {basis }}{ }^{5}\right)$. In the case of the two-electron repulsion integrals, the best known algorithms, which incorporate the full eightfold permutational symmetry, require $25 / 24 \mathrm{~N}_{\text {basis }}^{5}$ multiplications (Saunders 1983). The entire set of transformed integrals is not always required. For instance, MCSCF wave function optimization, some formulations of MRSDCI and second-order perturbation theory require only a subset of the integrals with $0-, 1-$, and 2 -external indices. The following discussion concentrates on integral transformations, but is generally applicable (e.g., transformation of MO density matrices to the $\mathrm{AO}$ basis).

Whiteside et al. (1987) used a 32-node Intel iPSC-2 and assumed AO integrals partitioned by charge distributions. The transformation algorithm used required $5 / 2 \mathrm{~N}_{\text {basis }}{ }^{5}$ total arithmetic operations. Small test cases realized speedups between 12 and 31 on 32 nodes. An algorithm requiring $7 / 2 \mathrm{~N}_{\text {basis }}{ }^{5}$ operations was used by Covick and Sando (1990). The communications time was reduced by a factor of two on a 2-D mesh, and a factor of $\mathrm{N}_{\text {basis }}$ on a hypercube, compared to the Whiteside hypercube implementation. A variation of this algorithm, with memory requirements of $\mathrm{N}_{\text {basis }}{ }^{4} / 4$, was implemented by Harrison (1992) on the Intel Touchstone Delta. The computation was overlapped with the intermediate transposition and a performance of 13 GLOPS was measured for a 200 basis function test case. The required presort was not considered. Limaye and Gadre (1994) discussed several possible algorithms and adopted an integral driven approach which required $\mathrm{O}\left(\mathrm{N}_{\text {basis }}{ }^{2}\right)$ local memory. The AO integrals were sorted and written to disk, for subsequent dynamically balanced transformation using an inhomogeneous 128 processor Transputer cluster. Dupuis (1992) discusses three partial transformation algorithms, two of which are appropriate for parallel implementation. Windus et al. (1994) use the second algorithm in a parallel MCSCF implementation in which the entire AO integral list is replicated on local disks on each node. Timings are reported on a cluster of 1 to 5 workstations.

\subsubsection{Configuration Interaction}

In the configuration interaction (CI) method, the many-electron wave function is expanded in Slater determinants or spin-adapted configuration state functions (CSF), usually constructed from othonormal orbitals. Conventional CI methods explicitly construct the Hamiltonian matrix and apply an iterative eigenvalue method. Most algorithms for the solution of the eigenvalue problem require the formation of matrix-vector products $\underline{w}=\mathrm{H} \underline{v}$ for a set of intermediate vectors $\underline{v}$. This feature is exploited in integral-driven direct-CI methods which avoid the explicit construction and storage of the potentially large matrix, H. For large-scale wavefunction expansions, the computation of these matrix-vector products dominate the overall procedure. Conventional and selected-CI methods are straightforwardly parallelized. The Hamiltonian matrix elements may be independently computed and stored on disk or in memory. A replicated data approach may be adopted for the matrix-vector products since the $\mathrm{Cl}$ expansion is typically only $\mathrm{O}\left(10^{3}-10^{5}\right)$. Selection of configurations based on the interaction with a reference set can be parallelized over the set of trial configurations. Implementations are reported by several authors (e.g., Honju 1991). 


\subsubsection{Full-Configuration Interaction Methods}

The full-CI wave function includes all possible CSFs of the appropriate $S^{2}$ and $S_{z}$ (or determinants $S_{z}$ ) spin quantum numbers. Full-CI is the exact solution of the nonrelativistic Schrodinger equation in the chosen 1-particle basis, and the energy is invariant to orbital rotations. The length of the full-CI expansion grows very rapidly with the number of electrons and molecular orbitals, and consequently, full-CI wave functions can be computed only for relatively small systems. Insertion of a resolution of the identity in the N-electron full-CI space allows (Knowles 1984; Siegbahn 1984) the expensive twoelectron component of the Hamiltonian matrix-vector product to be expressed such that the majority of arithmetic operations occur in a matrix-matrix product of integrals with an intermediate matrix D. Projecting onto the $\mathrm{N}-2$ particle space greatly reduces the number of intermediate states and, therefore, the total operation count. A spin-free formulation of the $\underline{\mathrm{N}-2}$ projection method was adopted by Harrison and Stahlberg (1993) and implemented for MPPs. This algorithm had 12-fold fewer floatingpoint operations than the previous best determinant-based algorithm (Olsen 1988) due to the reduced number of intermediate states, but the increased complexity of the coupling coefficients reduced this to an overall approximate sixfold improvement. An object-oriented-like approach provided a portable interface for all processes to the shared $\underline{w}$ and $\underline{v} \mathrm{CI}$ vectors, which were asynchronously accessible by all processes. The matrix vector product was parallelized at a coarse grain over blocks of intermediate states, $\underline{\mathrm{K}}$, with the MO integrals replicated within each processor. Implementations of the $\mathrm{CI}$ vector objects used message passing on the Intel Touchstone Delta, compiler directives on the Alliant and Cray computers, and also explicit shared-memory programming on the Alliant. Calculations on the Intel Delta sustained in excess of four GFLOPS on 512 processors. Limited memory precluded performing calculations on few processors, but efficiency was estimated as greater than $50 \%$. The largest calculation was on methane in a $3 \mathrm{~s} 2 \mathrm{pld} / 2 \mathrm{~s} 1 \mathrm{p}$ basis with a frozen core, and included 95 million CSFs in $\mathrm{C}_{2 v}$ symmetry (418 million determinants). The calculation was possible only with extensive use of data compression and a modified iterative diagonalization method. Although an out-of-core version was implemented, $\mathrm{I} / \mathrm{O}$ on the Delta was too slow to permit yet larger calculations.

\subsubsection{Multireference Single and Double Configuration Interaction}

Multireference single- and double-excitation CI (MRSDCI) is currently the method of choice for studying chemical reactions. The multireference wave function is required to describe accurately the bond-breaking, bond-formation, and spin-recoupling effects that occur in the description of global molecular potential energy surfaces. Extensions of accurate MRSDCI methodology to molecular systems larger than 10 atoms requires enormous resources, and such calculations are an ideal match for the potential offered by parallel computers. The COLUMBUS MRSDCI program (Shepard 1988) has been parallelized using message passing. Pairs of segments of $\underline{w}$ and $\underline{v}$ are dynamically assigned to processes which read the segments from disk as necessary and compute all interactions. The 3- and 4external molecular integrals are processed $\mathrm{N}_{\text {segment }} / 2$ times, whereas the 2-external integrals are potentially processed for every segment pair. Again, the integral file and current $\underline{v}$ file are shared between processors, with each process having a local file for contributions to $\underline{w}$. The sequential program stored the partial coupling coefficients on a file and read them for each segment pair. However, good parallel load-balancing requires many small CI segments, each of which use only a small fraction of the coupling coefficients. This overhead is eliminated by recomputing the internal coupling coefficient factors on-the-fly as needed, for which the graphical unitary group approach is ideal. A speedup of 7.2 on an eight processor CRAY Y-MP was achieved for the matrix-vector product, with an overall speedup of 5.9. On the Intel iPSC-i860, the asymptotic speedup was limited 
to 3.4 by slow $\mathrm{I} / \mathrm{O}$, particularly for the 2-external integrals, $\underline{w}$ and $\underline{v}$. Subsequent work by Lischka et al. (1994) kept copies of as many arrays as possible on a local virtual disk in the central memory of each processor. Much improved efficiency was achieved only for up to 40 iPSC-i860 processors because this was only possible for small problems and available parallelism was exhausted. A shared virtual disk was implemented using the global array tools described above for the distributed SCF program. Input/Output intensive quantities were placed in the global arrays, and a single copy of $\underline{w}$ was similarly shared. A speed-up factor of 36 on 40 modes of the Intel Touchstone Delta was achieved in an MRSDCI calculation on $\mathrm{CH}_{3}$ in $\mathrm{C}_{2 v}$ symmetry and a cc-pVTZ basis $(624,334 \mathrm{CSF}$ and 29 segments). Work in progress includes data-compression of $\underline{w}$ and $\underline{y}$, segmentation of the 0 - and 1external CSF space, and computation of the contributions of the 3- and 4-external MO integrals directly from the $\mathrm{AO}$ integrals.

\subsubsection{Many-Body Methods}

The size-extensive many-body methods may be regarded as arising from the cluster or exponential expansion of the wavefunction, $|\mathrm{u}\rangle=\mathrm{e}^{\mathrm{T}}|0\rangle$, where $\mathrm{T}=\mathrm{T}_{1}+\mathrm{T}_{2}+\mathrm{T}_{3}+\ldots \ldots+\mathrm{T}_{\mathrm{n}}$ is a sum of up to n-electron excitation operators. The reference function $(\mid 0>)$ is usually chosen as Hartree-Fock. Many-body perturbation theory (MBPT) sums all terms to a finite order of perturbation theory, while coupled-cluster (CC) methods sum selected terms to infinite order. In particular, MBPT(4) includes the contributions of single, double, triple, and quadruple excitations through fourth order. CCSD includes the contribution of single and double excitations through infinite order, and CCSD(T) is one of many possible formulations to include approximately the effect of triple excitations in coupled cluster (Bartlett 1989). The computational cost of MBPT(2) is $\mathrm{O}\left(\mathrm{N}_{\text {basis }}{ }^{4}\right)$ (excluding the necessary four-index transformation), while for CCSD and MBPT(4), without the triples, the cost is $\mathrm{O}\left(\mathrm{N}_{\text {basis }}{ }^{6}\right)$. The fourthorder contribution of the triples costs $\mathrm{O}\left(\mathrm{N}_{\text {basis }}{ }^{7}\right)$. However, whether formulated algebraically or diagrammatically, many-body theory results in well-structured, matrix-oriented equations that are very suitable for massively parallel computation.

The second-order perturbation theory (MBPT[2]) energy for a closed-shell system with canonical SCF orbitals is given by

$$
\begin{aligned}
E^{(2)}= & -i j a b \\
& (i a \mid j b)[2(i a \mid j b)-(i b \mid j a)] /\left(e_{a}+e_{b}-e_{i}-e_{j}\right) \\
& ={ }_{-i j a b} E^{(2)}{ }_{i j a b}
\end{aligned}
$$

with $i, j$ and $a, b$ corresponding to occupied and virtual orbitals, respectively, and $e_{p}$ an orbital energy. Computation of the above expression requires $\mathrm{O}\left(\mathrm{N}_{\mathrm{vir}}{ }^{2} \mathrm{~N}_{\mathrm{occ}}{ }^{2}\right)$ effort, and the overall computational effort is, therefore, dominated by the necessary four-index transformation. The parallelism in the MBPT(2) energy derives from the fact that the individual contributions to the energy $\mathrm{E}^{(2)} \mathrm{j \textrm {jab }}$ are independent. At the finest level, an individual contribution depends on only two distinct repulsion integrals, (ialjb) and (ib $\mid j a)$, and the four corresponding orbital energies. These two integrals must be brought together onto the same node, either by explicit communication or by tailoring the integral transformation program to produce the transformed integrals in this manner. The orbital energies are few in number and-may be replicated if necessary. Watts and Dupuis (1988) have given two parallel algorithms on the LCAP system, while Colvin et al. (1989) described two Intel hypercube-based parallel algorithms. Feyereisen implemented in DISCO a direct partial transformation of integrals for each occupied orbital, computing batches of $\mathrm{E}^{(2)}{ }_{\mathrm{i}}={ }_{\mathrm{j} \mathrm{jb}} \mathrm{E}^{(2)}{ }_{\mathrm{ijab}}$. The parallelism arose from distribution of the AO integral generation in 
the same fashion as done for the SCF (1993). The number of times the AO integrals must be computed was determined by available memory, since every node must hold $\mathrm{O}\left(\mathrm{N}_{\mathrm{occ}} \mathrm{N}_{\mathrm{virt}}{ }^{2}\right)$ integrals for each index $\underline{i}$ in the batch. An AO-based parallel implementation of the SCEP approach of Pulay et al. to MBPT(2) is currently under development, again using the global array tools for distribution of data structures (Bernholdt and Harrison 1994). Three different distributions of the primary data (partially transformed integrals and $\underline{T}_{2}$ amplitudes) are used and the only communication required in the algorithm occurs during redistribution of the data. Due to the limited $1 / O$ bandwidth of MPPs in the near future, the entire calculation is performed in memory. The next stage of development will adopt an approach related to the local correlation method of Pulay (1993) to take advantage of the sparsity of integrals and excitation amplitudes. The memory requirements are $\mathrm{O}\left(\mathrm{N}_{\text {basis }}{ }^{2}\right)$ for local memory and $\mathrm{O}\left(\mathrm{N}_{\text {occ }}{ }^{2} \mathrm{~N}_{\text {basis }}{ }^{2}\right)$ for global memory.

Several groups have analyzed in detail parallel algorithms for the fourth-order MBPT triple excitation contribution, one such algorithm (due to Baker et al. 1991) being implemented on Cray computers using explicit shared-memory programming methods and dynamic load balancing. Almost peak performance of a CRAY X-MP/416 was attained, by emphasis on matrix-matrix multiplication and using many tasks to achieve good load balance. Subsequent work with the same algorithm using PVM on workstation clusters realized a best speedup of only 4.5 on eight processors due to the volume of communications and overhead of data migration. Watts and Dupuis (1989) had previously presented an algorithm with fourfold fewer operations than that due to Baker and discussed its parallel implementation. Rendell and Lee (1991) used an algorithm similar in spirit to that of Watts and Dupuis in their shared-memory vectorized $\operatorname{CCSD}(\mathrm{T})$ algorithm which was implemented on Cray computers using a parallel matrix-multiply routine and automatic compiler parallel optimizations. The long vector lengths and large matrices of their algorithm resulted in the excellent performance of 2.1 GFLOPS on a CRAY Y-MP/8 with a parallel efficiency of $90 \%$. Rendell et al. (1993) subsequently presented a new algorithm with much reduced $\mathrm{I} / \mathrm{O}$ that was suitable for massively parallel messagepassing machines. This strategy performed four times too many operations, but provided excellent parallel scaling, realizing an approximate speedup of 223 on 256 nodes of the Intel Touchstone Delta. Subsequent work on CCSD(T) by Rendell employed a distributed-data approach and improved their speedup to 234 on 256 Intel Delta processors.

A message passing implementation of the CCSD method reported by Rendell and co-workers (1992) had, as its objective, a semi-direct approach capable of treating systems with 500 virtual and 50 occupied orbitals. The equations were formulated so that local memory requirements were $\mathrm{O}\left(\mathrm{N}_{\mathrm{occ}}{ }^{2} \mathrm{~N}_{\text {virt }}\right)$ and that the contributions from molecular integrals with three and four virtual labels could be computed directly from the AO integrals. The memory constraint increased the computational cost of processing the four virtual integrals by fourfold. The poor I/O performance of Intel parallel computers forced replication of the amplitudes $\left(\mathrm{O}\left[\mathrm{N}_{\mathrm{occ}}{ }^{2} \mathrm{~N}_{\text {virt }}{ }^{2}\right]\right)$ and some integrals on each processor. Even so, the perfectly parallel I/O on the four-virtual integrals constrained overall speedup to six on 64 Intel Touchstone processors, compared with a CPU speedup of 48 . Subsequently, a distributed-data model was adopted using interrupt driven communication mechanisms. The goal of local memory requirements of only $\mathrm{O}\left(\mathrm{N}_{\text {occ }}{ }^{2} \mathrm{~N}_{\text {vir }}\right)$ was met by distributing all four index quantities with two virtual indices. Furthermore, the flexibility offered by the programming model enabled the computational cost to be reduced relative to the message passing algorithm. A calculation with 2.2 million $\mathrm{T}_{2}$ amplitudes was presented which would have been impossible with the replicated data method. Most recently, the global array tools of Harrison et al. have been incorporated into Rendell's CCSD(T) program, resulting 
in a simplification of the program through the elimination of explicit message passing and interrupt handling. However, some optimizations using double buffered asynchronous communications were eliminated.

\subsubsection{Quantum Monte Carlo}

Quantum Monte Carlo (QMC) methods attempt to solve the Schrödinger equation with direct numerical simulation. Diffusion Quantum Monte Carlo (DQMC) is an example of a perfectly parallel algorithm, which parallelizes at the coarse grain level with essentially zero communications overhead; in addition, it is also readily vectorized. The Green's Function Monte Carlo (GFMC) methods typically display much increased branching compared to DQMC, implying that much greater attention must be paid to load balancing.

Anderson et al. (1993), using first the SIMD CM-2 and then the MIMD CM-5, applied the exact GFMC algorithms of Anderson to provide high-accuracy results for the four-particle hydrogen molecule and the helium dimer (1993). The SIMD program mapped each independent point to a virtual processor, and was complicated by the branching and the need to dynamically allocate and deallocate processors. It was also necessary to leave approximately $10 \%$ of memory free to avoid exhausting memory while branching. A performance of 1.5 GFLOP on a 65,536 processor CM-2 was claimed. Their best calculation required 60 hours on a 32,768 processor CM-2. The MIMD program adopted a replicated-data, message-passing approach and required minimal changes to the FORTRAN sequential program.

Several algorithms for parallelizing the exact fermion GFMC method of Kalos (1992) have been discussed by Zhang and Kalos (1993). Their adopted strategy replicated information about the entire population on each processor in order to support the cancellation algorithm. Independent threads of execution were assigned unique random number seeds and deterministically assigned to specific groups of walkers. Using PVM on a network of ten IBM RS600 processors an efficiency of $80 \%$ was obtained in calculations on the ground state of the beryllium and nitrogen atoms.

\subsubsection{Applications of Parallel Computers}

Parallel computers are now a viable resource for quantum chemistry computations. This assertion is most vividly demonstrated by the increasing volume of work that use such machines. One can point at several calculations that, when they were performed, were state-of-the-art in large part because the use of parallel computers provided computational power and capacity of which exceeded that available elsewhere. Such calculations include the GFMC results of Anderson (1993), the large molecule property calculations of Dupuis et al. (1988), the full-CI results on methane due to Harrison (1993), and the large molecule SCF work of Sim (1987).

More impressive still is the extensive use now being made of smaller parallel computers and workstation clusters to provide cost-effective computing. The following must be just a very small sample since many of these works make little or no mention that parallel computers were used and 
polarizabilities (Nichols 1993), selected CI and full-CI benchmarks for electron affinities (Kendall 1992), organometallic chemistry (Cundari and co-workers 1993), organic chemistry (Kraus 1993), CFC replacement atmospheric lifetimes (Cooper 1993), and gas phase reaction rates (Buttar 1994).

\subsection{Quantum Reactive and Inelastic Dynamics}

With the advent of massively parallel processors, we are on the verge of a revolution in the scale of problems that can be solved by accurate quantum dynamics. Such methods achieve accurate, converged solutions (i.e., those do not change with the size or the nature of the basis set). While progress in method development lies somewhat behind electronic structure because of the greater complexity of time-dependent phenomena, recent progress on MPPs has been significant. Only a few groups are actively involved in parallel code development, but a rapid proliferation of effort in this field is expected. The range of problems currently amenable to study is now constrained by the limited power of vector supercomputing, and access to higher performance MPP systems is viewed as an essential requirement for continuing progress.

The determination of the cross sections and the rates of chemical reactions is a central goal of theoretical chemistry. Quantum dynamics is important both scientifically and technologically, and establishes the relationship between structure (potential energy surfaces) and reactivity (cross sections and rates). Sensitivity analysis permits a determination of the regions of potential energy surface which are important for dynamics and can help guide $a b$ initio electronic structure calculations that refine the surface. In some cases, the inversion of measurements to the potential energy surface via quantum dynamics can lead to a calibration of the accuracy of various electronic structure techniques.

Benchmark calculations, which at present are limited to relatively small systems, can be used for determining the validity of approximate methods to be applied to much larger systems. In particular, they permit testing the range and conditions of validity of classical trajectory and reduced dimensionality methods. The need for such methods is demonstrated by the fact that complex chemical systems involve hundreds of reactions whose cross sections and rates are not known and can be difficult to measure. The concepts underlying chemical reactivity are essential to expand the measured or calculated data base to the size necessary to do technologically significant simulations. As computational methods and hardware improve, a larger fraction of the data base can be generated theoretically, perhaps in a more cost effective manner than experiments.

The determination of cross sections and relaxation rates for inelastic processes is also important. For example, the stabilization step of addition reactions involves inelastic scattering of hyperthermal reactants, a phenomena not readily amenable to experiment because of the difficulty of preparing the reactants in such highly energetic conditions. Calculations can play a critical role in understanding the special features of this process.

\subsubsection{Quantum Dynamical Methods}

There are three basic quantum dynamical methods - time independent propagation and variational methods, and time dependent wave packet techniques - used in the interpretation of scattering results. All of these methods are very computationally intensive and exhibit differing levels of complexity in 
parallelization. Current experience, primarily on triatomics, shows that the methods are more CPU bound than I/O bound; they are also memory intensive, with the data structures involved so large that they must be distributed. The latter constraint usually makes present message passing implementations machine dependent.

The methods of quantum dynamics are dominated by basic linear algebra routines. Crucial routines in some implementations of the above methods include matrix inversion (propagation), matrix eigensolution (in variational methods), and FFTs (in wave packet studies). Although the widespread use of these types of linear algebra routines typically leads to their incorporation in single-node libraries of most MPP systems, much work is required to establish scalable distributed data implementations. Reduced dimensionality methods and inelastic and reactive scattering theories employ the same fundamental techniques. The only major difference is that for a given number of atoms, the number of scattering channels that must be considered is much smaller for inelastic than for reactive systems. Consequently, for a given computational expense, a larger numbers of atoms can be considered by a reduced dimensionality method or in an inelastic collision.

Relatively few groups are, at present, developing parallel implementations of quantum dynamics and reactive scattering applications. Scalable propagation codes for MIMD architectures using the log derivative method have been implemented by both Kuppermann and Lagana, while parallel electron scattering codes are under development by McKoy and coworkers. Progress in these areas and the dissemination of the associated techniques and expertise should be encouraged.

The input required in performing scattering calculations of a single electronic state is an accurate potential energy surface. With the advent of improved quantum dynamic methods and their implementation on parallel architectures, the demand for such surfaces is expected to increase dramatically. The rate-of-generation of these surfaces must clearly be accelerated, requiring the development of efficient correlated capabilities in parallel electronic structure codes, targeted to triatomic and tetratomic systems. Furthermore, the representation of the directly calculated energies at discrete points as an analytical surface involves a complex fitting procedure which, to date, has been unsystematic and tedious and must now be automated.

Chemical reactions occurring on more than a single electronic potential surface are also of fundamental and growing interest; all radical and electronically excited chemistry, for example, involves multiple surfaces. The input now required for performing scattering calculations are the multiple energy surfaces and their couplings (which are of the same dimensionality as the surfaces). Furthermore, for dynamics, the diabatic representation of these surfaces is the most convenient, since the corresponding coupling elements vary more smoothly with the geometry of the system. This implies an additional diabatization step once the adiabatic energies and couplings are obtained. The fitting problem is more acute here, given the increase in the number of surfaces and the different topological features of the surface couplings.

\subsubsection{Future Developments}

The promise of Teraflop computing opens up a new level of synergism between electronic structure, quantum dynamics, and experiment for systems comprising a few atoms. Consider, for example, the computational expense associated with a four atom reactive potential energy function. 
Given six degrees of freedom, and assuming that approximately 10 points per degree of freedom are needed to yield a surface of chemical accuracy, then electronic structure calculations at some $10^{6}$ geometries will be required. In order to minimize the corresponding computational effort, it is crucial to perform scattering calculations limited to a small number of total angular momenta and energies to guide, via sensitivity analysis, the selection of geometries and the accuracy (and hence cost) of the former calculations. This coupling, and associated gradient calculations at each geometry, may lead to an order of magnitude reduction in the number of geometries at which high accuracy calculations must be performed. Therefore, if the energies at $10^{3}$ geometries could be generated in an hour on an MPP, and if quantum dynamics calculations for limited energies and angular momenta could be performed at the same rate, then it would be possible to tightly couple the dynamics and electronic structure on a daily basis. These estimates of time are realistic if, and only if, centralized teraflop machines become available. With this level of coupling, the study of reaction systems of four or less atoms would enter a new phase. Experiments and calculations would themselves become tightly coupled, permitting calculations to guide experiments in real time, and vice-versa.

\subsection{Molecular Dynamics}

Computer simulation by Molecular Dynamics (MD) is one of the most powerful techniques of modern physical science. It provides arguably one of the best means of addressing fundamental questions concerning the dynamical and structural behavior of bulk materials and plays a key role in our understanding of microscopic processes in physical systems. It is now widely used in materials science, chemistry, biology, and physics. The inherently parallel nature of MD computations has been widely discussed and reviewed in the literature, with the rich degree of intrinsic parallelism that characterizes problems in molecular simulation at several different levels now well-established. Three basic strategies have been adopted in the migration to parallel processing, involving the mapping onto processors of particles, of interactions, or of regions of space.

We structure this overview of state-of-the-art developments in MD software by considering initially the computational demands of the discipline, followed by a summary of parallel algorithms, with an outline provided of the methods employed in the computation of both short- and long-range forces, and the increasing use of multiple-time scale methods. The issue of scalability is also considered where, based on the preceding discussion, we pinpoint the emergence of algorithms capable of exploiting MPP machines with hundreds of processors in efficient fashion. Finally, we review the current parallel status of those simulation packages widely used within the simulation community - CHARMM, DISCOVER, GROMOS, and AMBER. All have been migrated to parallel hardware using a variety of replicated data schemes, with good efficiency achieved in some cases for up to a few dozen processors. Although this level of performance clearly provides a reasonable tool for ongoing application studies, the goal of truly scalable parallelism is unlikely to be realized based on the implementation schemes adopted to date.

\subsubsection{Computational Demands and Goals of Molecular Dynamics Simulations}

Each of the $\mathrm{N}$ atoms or molecules in an MD simulation is treated as a point mass, and Newtons equations are integrated to compute their motion. A range of useful information, both microscopic and macroscopic, can be obtained from the motion of the ensemble of atoms, including transport 
coefficients, phase diagrams, and structural or conformational properties. Molecular Dynamics simulations for complex physical systems often involve large system sizes ranging from $10^{4}$ to $10^{6}$ particles. A crucial feature of a given simulation is the form of the $\mathrm{N}$-body interparticle interaction energy, $\mathrm{V}$, which is typically expressed as a combination of one-body, two-body, three-body potentials etc., with the two-body or pairwise interactions usually the most important terms. Regarding the time evolution of particles, it is often vital to investigate this time development over hundreds of picoseconds. Since the time increment needed to solve Newtons equations is on the order of a few femtoseconds, given the need in liquids and solids to accurately model vibrational motion, MD simulations will typically involve solving Newtons equations for $10^{4}-10^{6}$ time intervals to simulate even picoseconds of "real" time. These computational demands highlight the difficulty of simulating large systems over long intervals of time on sequential machines, with hours of CPU time required on machines such as the CRAY C90 to perform picosecond simulations for $10^{5}$ atom systems.

When assessing the algorithmic developments required to achieve the computational goals of MD simulations, there are two quite distinct requirements. The first is the development of algorithms which are effective for a small number of atoms (typically $N<1000$ ), for the aim in any simulation must be to model, to a sufficient level of accuracy, the required physical effects with the smallest possible value of $\mathrm{N}$, thereby performing each time step as rapidly as possible. The vast majority of MD simulations are carried out on systems with between several hundred to a few thousand atoms, and it is often more useful to be able to perform a $10^{5}$ timestep simulation of a 1,000 atom system faster than a $10^{3}$ timestep simulation of a 100,000 atom system, although given an $\mathrm{O}(\mathrm{N})$ scaling, the computational effort involved is identical in each case.

The second requirement, in the area of large MD simulations, is the ability to develop truly scalable algorithms. The development of such algorithms that scale optimally with both the number of atoms and the number of processors will leave the simulation community ideally positioned to exploit the next generation of more powerful parallel machines.

In the drive to optimize use of available hardware to enable more robust simulations through increasing system size and simulation length, the optimization of MD algorithms for vector hardware has been extensively discussed in the literature, with considerable attention paid to the task of optimizing efficiency. We provide below some background to this effort, since much of the work impacts on subsequent parallel implementations.

In short-range $\mathrm{MD}$, the computational effort per timestep scales linearly as $\mathrm{N}$, the number of atoms, with the summation restricted to atoms within some small region surrounding atom $i$, and implemented using a cut off distance outside of which all interactions are ignored. The key to efficient force evaluation stems from knowing which neighboring atoms must be tested for possible interactions, with two basic methods used to derive this information on serial and vector machines, neighbor lists and the link-cell method. In the former method a list of the non-bonded "neighbors" of each atom (those within an extended cutoff) is maintained and typically updated every 10-100 timesteps. While efficient algorithms have been developed, the computational effort required to generate the list is not negligible; use of the list reduces the work of computing the nonbonded forces from order $\mathrm{N}^{2}$ to $\mathrm{nN}$, where $n<<N$. In the link-cell method all atoms are binned into 3-D cells with size of length $r_{c}$, so that the task of finding neighbors of a given atom reduces to checking 27 bins. In practice, the fastest MD algorithm on serial and vector machines uses a combination of the two methods, with optimal 
performance on vector machines demanding a detailed consideration of both loop constructs and data structures to ensure complete vectorization. The best implementation to date (Grest 1989) is a factor of 2 to 10 times faster than other vectorized algorithms.

\subsubsection{Parallel Algorithms in Molecular Dynamics}

Parallelization of MD simulations requires the distribution over processors of both the evaluation of pair interactions and the integration of particle motions. The force terms involved in integrating the set of coupled differential equations characteristic of any MD simulation are typically nonlinear functions of the distance between pairs of atoms and may be either long-range or short-range in nature.

Much of this work has been implemented on a range of parallel hardware, including both SIMD machines, such as the CM-2, and MIMD hardware with up to 64 processors. However, the number of scalable algorithms working effectively on MIMD machines with several hundred processors are very much in the minority and are only just beginning to appear. Another striking aspect of much of the work has been the widespread use of Transputer Arrays and, with it, use of the Occam language. Occam, the native language of the Transputer, is designed to support concurrent processing simply and effectively. Its supporters point to the success of the language in avoiding the cumbersome nature of having to program multiprocessors in standard sequential languages. Yet the future role of both Occam and the Transputer must be in doubt given the lack of portability and the failure to provide widespread access to the language on non-Transputer platforms, coupled with the excessive delay in producing the next generation Transputer (the T9000).

\subsubsection{Short-Range Forces}

All parallel algorithms that have been suggested to date have been based on two distinct methods for distributing the forces calculation evenly across all processors to extract maximum parallelism:

1. Each processor is assigned a predetermined set of force computations, involving a force decomposition of the workload that remains in effect during the simulation.

2. Each processor is assigned a portion of the physical simulation domain, involving a spatial decomposition of the workload.

Arguably the most definitive work on parallel algorithms for short-range MD is due to Plimpton and co-workers (1991), who presented three parallel algorithms suitable for MD models which can be difficult to parallelize efficiently - those with short-range forces where the neighbors of each atom change rapidly. In our discussion below, we group the various developments into these three categories:

- atom decomposition - Where each processor is assigned a subset of atoms.

- force decomposition - Where each processor is assigned a subset of interatomic forces to compute.

- spatial decomposition - Where each processor is assigned a fixed spatial region. 
Plimpton has described in some detail the implementation and testing of each algorithm on message-passing distributed-memory parallel machines. The algorithms were tested on a standard Lennard Jones (LJ) benchmark problem for system sizes ranging from 500 to $10,000,000$ atoms on three parallel supercomputers, the nCUBE2, Intel iPSC/860, and Intel Delta. Comparing the results to the fastest reported vectorized CRAY Y-MP and C90 algorithm showed that the current generation of parallel machines is competitive with conventional vector supercomputers even for small problems. For large problems, the spatial algorithm achieved parallel efficiencies of $90 \%$ and the Intel Delta performed about 30 times faster than a single Y-MP processor and 12 times faster than a single C90 processor.

Atom Decomposition. Atom-decomposition or 'replicated-data' methods are often used in MD simulations of molecular systems, the duplication of data involved making for the straightforward computation of additional three- and four-body force terms. Parallel implementations of CHARMM (Brooks 1992) and GROMOS (Clark 1991) are based on this technique. Each of the P processors is assigned a group of N/P atoms at the outset of the simulation and continue throughout to only compute forces on these atoms. The major advantage of this algorithm is simplicity; however, it does require global communication, as each processor must acquire coordinate and forces data held by other processors. Scaling as $\mathrm{N}$, independent of $\mathrm{P}$, this communication limits the number of processors that can be effectively used. In the $\mathrm{LJ}$ benchmark problem, communication costs begin to dominate by the time hundreds of processors are used, there being little speed-up when doubling the number of processors.

Force Decomposition. Two categories of force-decomposition algorithms have appeared in the literature: those that systolically cycle atom data around a ring, and those based on a force-matrix formalism. A variety of systolic loop methods have been implemented on both SIMD and MIMD processors. In essence, each particle is allocated a home processor which integrates its motion; in the evaluation of the interactions, the particle data circulate around the ring of processors in such a way that every particle meets every other particle, enabling all the pair interactions to be evaluated. Possible sources of inefficiency include imperfect load balancing and communication delays during the force evaluation, and communication delays in accumulating thermodynamic quantities. In both cases, the loss of efficiency is only significant in the event that the number of processors approach the number of particles. Attention was drawn to the advantage of asynchronous communications in reducing communication delays during the force evaluation. Modifications to the systolic loop method include systolic replication (Fincham 1991) and replicated systolic loop (Windemuth 1992).

The very efficient nature of systolic loop programs in MD simulations of liquid systems relies on the number of molecules simulated exceeding the number of processors used. Simulations of large flexible molecules often do not meet this condition, requiring the three- and four-body terms used to model chemical bonds within a molecule to be distributed over several processors. The systolic loop methods have generalized to accommodate such systems, providing a number of computer programs for the simulation of protein dynamics (Raine 1991).

The force-decomposition algorithms due to Plimpton and co-workers are based on a blockdecomposition of the force matrix rather than a row-wise decomposition as used in the atomdecomposition algorithm. This block-decomposition strategy means that each processor requires only $\mathrm{O}(\mathrm{N} / \mathrm{P})$ information to perform its computations, reducing the memory and communication costs by a factor of _ $P$ versus the atom-decomposition algorithms. Additional advantages of these algorithms include the ability to use the same communication utilities required in atom-decomposition, and the 
ability to obtain optimal performance in the absence of geometric information. In the LJ benchmark problem, the force-decomposition algorithm continues to speedup, even when hundreds of processors were used; in contrast to the atom decomposition results, an increase of $30 \%$ in speedup was observed when the number of processors was doubled.

Spatial Decomposition. In spatial-decomposition or geometric methods, the physical simulation domain is subdivided into small 3-D boxes, one for each processor. Each processor computes forces on and updates the positions and velocities of all atoms within its box at each timestep, with atoms reassigned to new processors as they move through the physical domain. Since a processor need only know positions of atoms in nearby boxes in order to compute forces on its atoms, the communication required in the spatial decomposition is local in nature, in contrast to the global communications required in the atom- and force-decomposition methods.

The suitability of spatial-decomposition methods for very large MD simulations is reflected in the large body of research reported, to date, across a wide range of parallel hardware. Algorithm design, programming issues, timing experiments, and scaling projections have been widely presented, revealing that the issue of load-balancing is an important consideration in any spatial-decomposition algorithm. Typical simulations reported to date include the feasibility studies of Rapaport (1991) for both two-dimensional (with 4 million particles) and three-dimensional systems (with 2 million particles) on a hypercube array containing 64 processors. The reported performance was found to be comparable to that of the latest vector supercomputers.

In the large $N$ limit, spatial decomposition should achieve optimal $\mathrm{O}(\mathrm{N} / \mathrm{P})$ scaling and is clearly the fastest algorithm, assuming that good load-balance is also achievable. An efficient implementation is clearly more complex than that encountered with either the atom- or force-decomposition methods. The spatial-decomposition algorithms, due to Plimpton (1991), rely on a communication scheme that involves extra coding and bookkeeping to create messages and access data received from neighboring boxes. Integrating these algorithms into an existing serial code would almost certainly involve a substantial reworking of data structures and code. Timings presented by Plimpton show that a spatialdecomposition algorithm had a parallel efficiency of $76 \%$ on 1,024 processors of the nCUBE2 and $80 \%$ on 512 processors of the Intel Delta for a million-atom simulation; larger simulations were shown to approach roughly a $90 \%$ efficiency.

2.3.2.2 Long-Range Forces. Coulombic interactions in an ionic solid or biological system are typical of the long-range forces encountered in MD simulations and, in general, involve each atom interacting with all others. Direct computation of these forces scales as $\mathrm{N}^{2}$, and becomes computationally prohibitive for large $\mathrm{N}$. A variety of approximate methods have been developed to overcome this bottleneck, including particle-mesh algorithms, which scale as $f(M) N$, where $M$ is the number of mesh points, hierarchical methods, which scale as $N \log (\mathrm{N})$, and fast-multipole methods which, grouping particles distant from the one under consideration, scale as $\mathrm{N}$. Recent parallel implementations of these methods have certainly acted to increase their range of applicability.

Kalia and co-workers (1993) have described atom- and spatial-decomposition algorithms for MD simulations of bulk systems with Coulomb forces, in which the conditionally convergent series for the $1 / \mathrm{r}$ interaction is treated by the Ewald method of summation. Testing these algorithms on an Intel iPSC/860 system revealed comparable execution rates, with the computation time dominating that associated with communications. 
One of the most promising methods for removing the bottleneck of the $\mathrm{N}^{2}$ computations, implicit in long-range interactions, is the Cell Multipole Method (CMM). Goddard and co-workers (1992) have described the method which scales linearly with $\mathrm{N}$ and requires only modest memory, and demonstrated the feasibility of the approach through a series of systematic calculations on realistic polymer systems (with up to 1.2 million atoms). The method treats a class of interactions of the form $\mathrm{q}_{\mathrm{i}} \mathrm{q}_{\mathrm{j}} / \mathrm{r}^{\mathrm{pij}}$ which includes Coulomb $(\mathrm{p}=1)$, London dispersion $(\mathrm{p}=6)$, or shielded Coulomb $(p=2)$ interactions, and becomes faster than the exact method for systems of 300 atoms; for a 1.2 millionatom polymer, it is 2,377 times faster. The method has been shown to be well-suited to highly parallel and vector computers.

\subsubsection{Multiple-Timescale Molecular Dynamics Methods}

Notwithstanding the algorithmic developments described above, the problem remains, in macromolecular structure at least, that the current time and spatial scales are inappropriate for the target physical questions. High-resolution experimental techniques and quantum-mechanical methods are limited and, from MD simulations, information spanning several order of magnitude beyond the currently accessible picosecond-nanosecond time frame is needed. While, in theory, the dynamic picture generated by MD should reveal many local energy minima as well as possible transition pathways, numerical stability considerations restrict the time step to the femtosecond range; this limits the total length of the trajectory that can be generated, and consequently, the scope of molecular motions that can be captured.

Algorithm development aimed at allowing longer timesteps to be taken on average, by performing work at staggered times on different length scales, lies at the heart of multiple-timescale MD methods. These schemes attempt to incorporate long-range effects while avoiding true long-range force computation; implemented by a hierarchy of neighbor lists, which store information for the different length scales, they typically involve force computation using very short-range information in the smallest timesteps.

Slick and Olson (1993) developed such an algorithm which permits larger time steps. The implicit Euler integration scheme is combined with the Langevin dynamics formulation, which contains frictional and random forces in addition to the systematic force; by choosing the frictional damping constant appropriately, it was shown that the high-frequency vibrational modes can be effectively damped. In particular, this damping can be set to mimic a quantum-mechanical like discrimination among the various vibrational modes. The minimization of the nonlinear function required at every time step in this implementation was carried out with a large-scale truncated Newton algorithm tailored for potential energy functions.

Nakano, Vashishta, and Kalia (1993) described the implementation of a parallel multiple-time step algorithm with three-body interactions. A domain-decomposition algorithm was used for particles interacting via two- and three-body potentials, employing the linked-cell-list method and separable three-body force calculation. The force calculation was accelerated by the multiple-time-step (MTS) method. For a 1.54 million $\mathrm{SiO}_{2}$ system, the $\mathrm{MD}$ program ran at a speed of 660 time steps per hour (1100 steps per hour without the three-body interactions) on a 64 -node Intel iPSC/860. The parallel 
algorithm was highly efficient (parallel efficiency $=0.973$ ) as it involved only $3 \%$ communication overhead. Using the second derivatives of the potential energy, the conjugate-gradient search for a local minimum underlying an MD configuration was accelerated by a factor of 13 .

Schulten (1992) outlined the development of a multiple-time scale approximation (distance class algorithm) for the evaluation of nonbonded interactions, as well as the Fast Multipole Expansion (FME). The efficiency of the FME was demonstrated, outperforming the direct evaluation of Coulomb forces for 5,000 atoms by a large margin, and showed, for systems of up to 24,000 atoms, a linear dependence on atom number.

\subsubsection{Parallel Simulation Packages}

Classified as "intermediate" sized codes within the chemistry community ( 100K-200K lines), simulation packages, such as CHARMM, DISCOVER, GROMOS and AMBER, have probably made the largest strides in exploiting the potential of parallelism. All have been migrated to parallel hardware employing replicated data schemes.

Recent work at the NIH to modify CHARMM has resulted in efficient performance on any MIMD system from the Intel Delta Touchstone at Caltech, to a simple cluster of workstations. The parallel implementation (Brooks and Hodoscek 1993) has the following characteristics:

- a full-feature version - all features are functional (but not all parallel)

- provides efficient parallelization for molecular dynamics and energy minimization

- remains compatible with developmental CHARMM on sequential machines

- represents a completely general parallelization for MIMD machines.

In separate work, Lin and co-workers (1992) also reported the development of a parallel version of CHARMM for the Intel iPSC/860. The parallelization employed the methods of traditional partitioning to distribute the computational workload in the core stages of the $\mathrm{MD}$, namely, the calculation of forces, the integration of equations of motions, the correction of atomic coordinates by constraint, and the generation and update of the nonbonded list. The processors coordinated their activity using synchronous communication to disseminate intermediate values, the scheme adopted achieving proportional or near-proportional speedup in the distributed computation and significantly reducing the demand for computer memory, although communication costs grew substantially as the number of processors increased. The approach for distributing the nonbonded list did not require communication, and the 2-D SHAKE algorithm developed has overcome the difficulties in the parallelism of SHAKE imposed by its iterative nature.

A fully distributed data parallel version of CHARMM, using the PARTI (Parallel Automated Runtime Toolkit at ICASE) primitives, has been described by Saltz and co-workers (1991). These primitives allow the distribution of arrays across the local memory of multiple nodes, and permit the 
global addressing of these arrays even on truly distributed memory machines. While the replicated data version of Brooks and Hodoscek exhibited superior performance (by a factor of $\sim 3$ ), the size of addressable problems is limited by the replicated data implementation.

Parallelization of Kollman's AMBER's MD module, AMBERCUBE-MD, has realized a fully functional parallel version of the MD module of AMBER3a for distributed-memory hypercube computers, including parallelized procedures for the calculation of the long-range nonbonded Coulomb and $\mathrm{LJ}$ interactions, generation of the pairlist, intramolecular bond, angle, dihedral, 1-4 nonbonded interaction terms, coordinate restraints, and the SHAKE bond constraint algorithm (Kollman 1993). Results were presented for speedup and efficiency on a nCUBE machine, using up to 128 processors, as well as benchmarks for performance comparisons with the CRAY Y-MP and FPS 522 vector machines. A parallel implementation of the AMBER MD module by Sato and co-workers (1992) targeted the AP1000 distributed-memory parallel computer developed at Fujitsu Labs. Ltd. (consisting of up to 1,024 processor elements connected with 3 different networks). To obtain a higher degree of parallelism and a better load balance between processors, a particle division method was developed to randomly allocate particles to processors. Experiments showed that a problem with 41,095 atoms can be processed 226 times faster with a 512-processor AP1000 than by a single processor.

Finally, we would draw attention to a parallel macromolecular simulation package, DL_POLY, currently under development which incorporates many of the parallel developments due to Smith and co-workers (1994).

\subsubsection{Benchmarking and Molecular Dynamics}

One of the crucial requirements in objectively assessing the suitability of a variety of machine architectures in a given computational discipline is the availability of an appropriate set of standard benchmark codes. The simulation community appears to have addressed this issue, at least in the area of short-range $\mathrm{MD}$, through the widespread adoption of a specific benchmark problem that models atom interactions with a $\mathrm{LJ}$ potential. This problem has been used extensively by a variety of researchers in assessing the performance of parallel and vectorized algorithms.

One interesting feature to emerge from this benchmark is the markedly superior performance of MIMD versus SIMD machines in short-range MD simulations. The timings on a 32K-processor CM-2 are slower than a single-processor CRAY Y-MP, and much slower than the timings for the MIMD parallel algorithms given by Plimpton and those from the CM-5 and Fujitsu AP1000. This was attributed to the inefficiencies introduced on the CM-2 when having to code the construction and access of variable-length neighbor lists via indirect addressing. 


\subsection{Impediments to the Migration, Development, and Exploitation of Computational Chemistry Software on Massively Parallel Computers}

Notwithstanding the significant developments and progress made by the computational chemistry community in migrating software to parallel architectures, there can be little doubt that without a major acceleration in the current rate of progress, the community will not be positioned to fully exploit the emerging class of Terafiop computers. Below we continue to quantify this assertion, to analyze the root causes that have led to this situation, and to consider the steps required to facilitate the development of the wide spectrum of associated capabilities.

The impediments to the uptake and successful use of MPP in chemistry may be attributed to both shortand long-term causes. In the short term, we must identify and address those factors that create and exacerbate the evident inertia to rapid adoption of MPP technology, looking to reduce the effort involved in making the transition from workstations and conventional supercomputers. Contributing factors include the poor match between existing hardware, software tools and computational chemistry algorithms, the lack of routine access to MPPs for both development and production purposes, and the lack of appropriate chemical expertise within the present support infrastructure at existing supercomputer centers. The lack of integration of MPP resources with existing (e.g., desktop) functionality so essential to scientific productivity is clearly acting to slow down the rate of progress. This also constrains computational chemists effectively pursuing their scientific goals, given the substantial effort required in migrating chemistry applications to an MPP environment. Furthermore, there is a clear lack of awareness within the relevant communities of how best to interact in exploiting MPP technology; the chemical community appears unaware of how best to integrate with and acquire the necessary skills from the computer science community, while the limited integration of the academic and industrial communities certainly acts to constrain the industrial uptake of this technology.

In the longer term, it is clear that the current MPP revolution is just the first phase in the continued evolution of HPC architectures. Future machines will continue to provide challenges to the computational science community and to meet these will require a sustained investment in training, integration of the physical and computer science communities, and active participation of both communities with the vendors in their associated research and development (R\&D) efforts.

In developing these ideas, we identify below five distinct areas for consideration in addressing the issue of impediments. In Section 3.1, we consider the requirements in education and training, and in Section 3.2 discuss various issues relating to software and the crucial need for an Integrated Software Environment that is extended to incorporate MPP technology. We continue in Section 3.3 to consider the issues of integrating the relevant communities, and in Section 3.4 point to the need for a generally accepted suite of computational chemistry benchmarks. Finally, in Section 3.5, we consider requirements in the configuration and operation of MPP supercomputer centers. A summary of conclusions, together with the panel's recommendations arising from consideration of these five areas, are presented in Section 4.0. 


\subsection{Education and Training}

The overview of Section 2.0 clearly revealed the considerable software development effort associated with both the algorithmic and implementation advances required to fully exploit MPP systems across a variety of pressing Grand Challenge problems in chemistry. Collectively, the academic community, government laboratories, and industry simply do not have the required number of computational chemists specializing in large scale parallel computing that will enable this work to be completed in a timely fashion. In fact, it is the feeling of many panel members that the aggregate number of scientists dedicated to chemistry methods development for high-performance computing has been declining in the last decade. To deliver on the promise of massively-parallel computing will require a significantly increased emphasis on the education and support of methods development. While recent initiatives have undoubtedly invigorated such activities (e.g., in the context of several HPCC Grand Challenges), to achieve the necessary level of effort may well require a focused interagency program of support. Given the growing importance of computational chemistry to United States industry, it is reasonable to suppose that the industrial demand for scientists trained in this discipline will also rise, as well as the demand from the commercial chemistry software vendors, thus creating new industrial positions for some of these scientists.

Another major impediment to the migration of chemistry applications to MPP is the lack of knowledge on the part of many computational chemists of parallel methods (e.g., programming models), tools, and the capabilities of MPP systems. Furthermore, the number of computer scientists able to converse with and assist the computational chemistry community is limited to those with backgrounds in scientific computing. A major requirement identified by the panel in increasing the uptake of MPP technology is education in the methods used in large-scale scientific computing (i.e., parallel numerical algorithms, performance analysis, modern software engineering). New resources will be required to educate existing researchers in both computational chemistry and computer science, with the long-term constraint being the availability of undergraduate scientific computing courses. In the shorter term, an important activity is the development of integrated curricula for computational science at the graduate level. Professional education is largely accomplished through research collaborations, professional meetings and technical tutorials at conferences and workshops. The DOE, NSF, and NIH already support excellent programs in computational science education, and this panel recommends that these existing programs continue to be expanded.

The computational chemistry community. should be encouraged to take a more pro-active position to professional education, by organizing workshops and tutorials at professional meetings (similar to those at the ACM, SIAM, and IEEE events) that focus on the methods and tools to migrate codes to MPP systems. The computer science community needs to insure that numerical computation is given adequate support at the undergraduate, graduate, and postgraduate levels, with basic education in computer architecture and numerical analysis available to all computer science majors. It is also imperative that chemistry graduate students obtain basic education in software engineering and computer science.

A powerful message can be sent to the community through the demonstration of successful applications of MPP in solving significant problems. This demonstrator function is largely provided by the Grand Challenge projects currently funded as part of the HPCC programs. These projects aim to demonstrate the potential and capability of MPP, so that the application user community becomes 
involved and aware that MPP is vital to their long-term competitiveness. This panel is encouraged by the first steps taken by the agencies in supporting the Grand Challenge process as an effort to integrate the computer science and computational science communities.

\subsection{An Integrated Software Environment}

With software providing arguably one of the greatest areas of impediment, we focus our discussion below into three software related areas, namely that of compilers, languages and tools (Section 3.2.1), chemistry algorithms (Section 3.2.2) and architecture-related issues (Section 3.2.3).

\subsubsection{Compilers, Languages and Tools}

The existence of portable compilers and tools capable of generating efficient code on MPP platforms should make the process of programming these machines accessible to a wide spectrum of computational chemists. In practice, however, a two- or three-order of magnitude performance difference between optimized and naive multiprocessor code remains quite common, primitive compilation techniques and tools often negating any performance advantage a user might have hoped to gain by moving to a parallel supercomputer. It is unlikely that current high-end users will trade a large decrement in potential performance for ease of programming and portability; compilers and tools that significantly compromise performance are unlikely to be attractive to the computational science community.

\subsubsection{Computational Chemistry and Language Standards}

The process of defining FORTRAN data parallel language standards represents an on-going activity, with the High Performance Computing Forum having completed work on a language standard for High Performance FORTRAN (HPF). This standard was effectively defined as a "common denominator", containing features that a broad spectrum of users demanded and that vendors felt could be implemented with relative ease. This initial HPF definition did not, however, address a great many issues that are likely to be crucial to the efficient implementation of computational chemistry algorithms on MPPs. Specific examples of such features include those for supporting sparse, adaptive, and irregular problems, and those for supporting high performance $\mathrm{I} / \mathrm{O}$.

There appears to be widespread agreement among compiler researchers and vendors that the current HPF language standard will need to be broadened, and a group chartered to do this convened in early 1994. The results of ongoing compiler and tool research, performance studies associated with porting applications codes to MPPs and performance results obtained with the initial HPF are to be used in defining the extended version. Vendors and users appear to have significant interest in MPP versions of $\mathrm{C}$ and $\mathrm{C}++$, although we are unaware of any ongoing standardization process.

Compiler groups and many funding agencies are aware of the kinds of challenges that are posed by I/O intensive problems and by sparse, unstructured, and adaptive problems. The development of effective methods for dealing with the latter class of problems has been the focus of considerable recent research by many groups. Much less attention has been paid to challenges associated with support for I/O intensive problems. Once computer science research groups and/or vendors are able to 
demonstrate the effectiveness of runtime support, compiler transformations, and language features designed to deal with these challenges, vendors will begin to incorporate such features in their commercial offerings. It is only at this point that explicit provisions are likely to be made for sparse, irregular, and adaptive problems along with parallel $\mathrm{I} / \mathrm{O}$ in language standards definitions.

\subsubsection{Computational Chemistry and Computer Science}

Although not the central theme of this section (see Section 3.3), the very limited interaction between the computational chemistry community and computer science research groups has a major impact on the whole area of software development. Many computer science researchers choose to solve relatively simple model problems which may, or may not, capture the salient features of what computational chemists feel are their principal problems. Applications researchers are often reluctant to commit significant resources to collaborations with computer scientists due to the relatively long time it takes for such collaborations to pay off. A project that leads to the successful design of runtime support, compilation techniques, or language features may, in the long term, help the research community, but is unlikely to provide any short-term benefit to the collaborating computational chemist.

The bandwidth of this interaction needs to be dramatically improved. The unique aspects of computational chemistry problems should be taken into account in the ongoing systems software and language design process that is being carried out by vendors and research groups. Furthermore, incentives must be devised that encourage computer scientists and computational chemists to collaborate on the design of systems software.

The Grand Challenge awards provide a framework for this interaction, but the number of Grand Challenge teams is limited and we can expect only a very small number of these to be in the area of computational chemistry. Another way of increasing this interaction would be to fund the chemistry community to develop a set of templates and performance models which embody the patterns of computation, data access, and I/O seen in high-end chemistry codes. A third possibility would be to provide resources to encourage the collaboration of computer scientists and computational chemists in targeting key chemistry packages.

\subsubsection{Chemistry Algorithms}

Unlike many other application areas (e.g., CFD, QCD, structural engineering), the computational chemistry community makes routine use of a diverse set of algorithms, few of which are similar to the grid (regular or irregular) methods of these other communities. Furthermore, most chemistry algorithms are not trivially parallel in the manner of the monte carlo methods adopted for many problems in physics. Our algorithms are also not at all well represented in data parallel models (e.g., the current HPF draft standard) and, indeed, most current parallel chemistry codes adopt a MIMD task parallel model. Thus, unlike these other communities, computational chemistry has been unable to date to present a compact set of algorithms that may be used to characterize requirements, or even to enable the design of specialized machines.

With the advent of MPP, many researchers now believe (cf., the discussion at the preceding 'High Performance Computing in Chemistry' workshop) that to realize the true potential of these machines 
will require an examination of the basic algorithms, not only for their scaling with parallel architecture, but for their fundamental scaling with the size of system being simulated. This may be at the level of proposing new algorithms for the same model (e.g., scalable Hartree-Fock algorithms or fast summation methods for many particle simulations) but is also at the level of asking what theoretical model should be invoked to answer a specific question. Furthermore, few existing chemistry algorithms have been systematically examined for parallel scalability, most studies being incomplete or biased by imbalance in the hardware on which the studies were performed. There are already instances where the availability of MPP resources (measured by both memory and CPU power) have encouraged the adoption of innovative approaches that have either been not considered before, or perhaps discarded as being not feasible in previous computational environments.

A third, and related issue, is the extensive use now being made of computation in the chemistry industry. This provides an identifiable and long-term process by which basic research from national laboratories and universities has been taken up by industry with the growth of software companies providing the required quality, support, and added value (e.g., in the form of integration into the desktop and communication between different models). Thus, the software problem facing the chemistry community is not just that of the basic research community, which develops new theoretical methods or algorithms and applies them to areas of new science, but represents a more profound software engineering problem of migrating computationally intensive components of existing packages to MPP. Throughout this process, we must maintain integration with the rest of the extensive functionality required by the chemist (particularly in industry) to conduct his research, while keeping open the channels that transfer innovation from the basic researcher to the applied or industrial chemist.

As a community, we must identify more clearly algorithms and templates of code so as to influence the design of hardware and software tools. These requirements must be communicated to the vendors developing hardware and the computer scientists that are designing tools and environments.

One of the major factors constraining the MPP migration of chemistry software is the sheer volume of code and associated functionality. This is particularly true in the area of quantum chemistry (QC), where the seven or so widely used QC codes comprise some 3,000,000 lines of FORTRAN. At least half of these codes are now commercialized. Furthermore, much of this software provides the necessary building blocks for new theoretical developments. It is clear from the work conducted to date that the "rapidly gained" benefits arising from adopting a straightforward replicated-data approach to porting are limited. Such an approach might enable "todays" problems to be handled more costeffectively (at least in the Hartree-Fock and density functional areas), but the associated constraint in problem size precludes the ability to tackle the Grand Challenge scale of problem. The benefits of going beyond this naive approach have been demonstrated, although this still demands a significant amount of code restructuring. In general, it is fair to say that many of the MPP implementations reported to date have been based more on ease of migration than on the real scientific requirements.

This situation is certainly not helped by the absence of a portable programming paradigm and environment. To adopt or define an alternative to one of the emerging tools as a standard for computational chemistry would be a step forward. Again, the overwhelming insistence by computational chemists for performance, ahead of portability and robustness, does not help in the aim of generating portable software, since many of today's tools have high associated overheads. 
The aim here must be to evolve a chemistry environment (reminiscent, for example, of DCE) that will provide a seamless migration from, say, workstations, to workstation clusters, to MPP. This must lead to not only portable support for parallel applications, but also the means to integrate these codes into the base functionality of existing serial environments which either have not yet been, or do not need to be, ported to MPP. Input from the computer science community will be vital in achieving this goal of integration, and to target expressiveness, performance, scalability, and portability with production quality code.

\subsubsection{Architectural Requirements}

There are a number of major features characterizing present MPP machines that inhibit the rapid migration of chemistry codes, notably poor I/O systems, small node memories, large latencies for message passing, and the lack of explicit architectural support for global address spaces and shared memory. We briefly examine these issues in the subsections below.

\subsubsection{Poor Input/Output Systems}

Many algorithms in computational chemistry generate large intermediate files that demand hightransfer rates to temporary storage. Current parallel machines do not provide scalable highperformance I/O systems. The requirement here is one of balance, for increasing the aggregate CPU performance through an increase in the number of processing nodes must be matched by a comparable increase in $\mathrm{I} / \mathrm{O}$ performance. This problem is so severe that it has forced a major investment in the design of "direct" algorithms, whereby intermediate quantities are recomputed rather than stored on secondary storage for subsequent reuse. Certain methods that demand out-of-core storage are simply not feasible on today's MPP systems, without a major investment of resources associated with the design of direct techniques.

Furthermore, scalable, hierarchical storage systems are needed for the large amount of data generated; developers should look to provide data-base systems designed to handle this data.

\subsubsection{Small Node Memories}

The replicated data approach continues to characterize the majority of parallel Hartree-Fock and MD implementations. These codes are certainly scalable to a moderate number of processors, but require large amounts of node memory, since all of the associated dataset must be node resident. Several MPP systems currently on the market do not provide adequate memory sizes for these applications, severely constraining the size of system amenable to treatment. Systems that support virtual memory and local disks may ultimately improve this situation, but are clearly limited by the poor I/O performance discussed above.

\subsubsection{Large Message Passing Latencies}

Today's parallel machines have relatively large latencies for sending point-to-point messages. Since the scalability of MD and $a b$ initio codes is often determined by the ratio of computation to communication, speedups are, in many instances, limited to less than 100 for typical codes. These 
latencies must be reduced to enable acceptable performance on machines with thousands of processors. Some architectures, currently under development, incorporate various techniques for hiding latency and may prove to be well-suited for chemistry applications.

\subsubsection{Lack of Global Address Support}

Some chemistry algorithms (e.g., MRSDCI) are most naturally expressed in terms of operations on global data structures. However, most MPP hardware does not support global address spaces, relying instead on software implementations of shared memory. In addition, the lack of hardware coherency support for global shared memory forces the programmer to manage memory coherency, introducing unneeded complexity into the code. Hardware architectures that can support a globally shared memory (even if physically distributed) should improve the ability to migrate many chemistry codes.

The significant mismatch between the requirements of computational chemistry and the capabilities of the present generation of MPP hardware provides a clear pointer to the need for the chemistry community to develop a well-defined set of architectural requirements for its large variety of application areas. This should be done under the assumption that massively parallel computers will be the most powerful and most cost effective machines in the future, and will represent a major step forward in the aim of eliminating future architectural impediments. In developing these requirements, the community needs also to identify important benchmarks and computational kernels, providing the necessary reference points for performance.

A related issue is that hardware designers should be encouraged to consider the needs of computational chemistry in the development of new systems. This includes the programs funded by DARPA, the agency that supports much of the research and development for new systems.

\subsection{Integration of Communities}

Although chemistry accounts for a significant fraction of the computational resources devoted to research in industry, government laboratories, and universities, the chemistry community has not been driving the development of large-scale, high-performance computing systems to meet the requirements of their applications. To date, the dialog has primarily been between vendors and individual investigators or research projects; in a few instances, researchers have been able to maintain sustained dialog through ongoing interactions with vendor computational chemistry groups. Due to the sparse and fragmented nature of these interactions, the community has been unable to establish vendor focus on the key aspects of its application requirements. This general problem is exacerbated with MPP vendors, where the time pressures of a highly competitive, highly technological effort, and the small applications development staff associated with new product development and startup companies leave the vendors unable to adequately collect or collate information from the chemistry community.

Initiating a more pro-active interaction by the community with computer vendors will require the development of a collective consensus of key computer capabilities and software standards necessary for our work on MPPs. Only then can the vendors be provided with a coherent and consistent picture of how to make their machines best fit the chemistry market. A possible mechanism to achieve such a consensus would involve the establishment of focused workshops and work groups chartered to collect 
and condense the requirements of the community, to the point where they might be used to drive the development of computer hardware and software technology. These should include a definition of the computer architectures, capacities, and performance parameters that best support computational chemistry; an endorsement of software development tools, libraries, and environment standards; and the requirements in migration, portability, and interoperability.

It is also important to increase vendor involvement in computational chemistry conferences on high performance computing. Vendors should be encouraged to attend key meetings, so as to learn more about our requirements, and subsequently focus development efforts on these requirements.

While closer integration of the computational chemistry and vendor communities will undoubtedly prove beneficial, there is perhaps greater benefit to be gained from more effective interactions with the computer science community. It is increasingly apparent that the adoption of modularity and modern software practices is vital to obtain substantial performance improvements of chemistry applications on high-performance computing hardware. The computer science community has gained substantial experience in the development of nonprocedural based programming methods (e.g., object-oriented paradigms) and in the performance analysis of applications on various hardware configurations. Extensive use of traditional supercomputing resources by computational chemists has led to an in-depth understanding of the algorithmic issues and performance of chemistry applications. These two communities must merge their respective expertise if the potential of Teraflop computing is to be realized.

Computational chemistry applications have positively impacted many industrial programs by the uptake of theoretical models validated by both the academic and industrial communities. The latter has often sought the expertise of academic chemists in the pursuit of their research and development activities. Unfortunately, the detailed requirements of industrial researchers cannot, in most cases, be widely publicized, given the position of commercial competitiveness and security. It seems unlikely that industry will move to high-performance MPP hardware until "turnkey" application software is available, yet the commercial chemistry application developers are unlikely to target a market that is currently as small as that of MPP. It is important for industry to establish well-defined, but general, requirements and make them better known to the academic community (university and national laboratory researchers), since the first usable software for MPPs will almost certainly be produced by the latter.

\subsection{Benchmarks}

There has been much publicity from MPP vendors concerning "peak performance". Yet, for the majority of computational chemistry applications, such performance has rarely been achieved, let alone sustained, while the ratio between observed and peak performance is typically far smaller that obtained from conventional vector supercomputers. This may, in part, be attributed to the poor balance of present generation MPP machines for computational chemistry, but clearly the community should have access to a widely accepted set of benchmarking capabilities so as to be able to continually monitor all aspects of performance associated with these machines. 
The chemistry community should follow the example set within computational fluid dynamics, where MPP vendors have been influenced by the availability of a generally accepted suite of benchmark programs (e.g., the NAS kernels). These codes serve to allow the community to compare the performance of MPP machines with each other and with existing conventional supercomputers, define the requirements of the application area for MPP, provide an application specific focus for hardware and software developers, and act to raise the profile of the application area.

\subsection{Configuration and Operation of Massively Parallel Processor Centers}

A crucial requirement for chemistry users to be able to successfully exploit present HPCC-MPP centers is that due consideration be given to the special needs of the community. These include the provision of adequate secondary (disk) and tertiary storage for both temporary and permanent (several weeks) data (e.g., trace files from long simulations which require repeated analysis); fast networking to enable desktop visualization of results; large memory configurations to support replicated data algorithms which, while not intrinsically scalable, provide rapid demonstration of the power of MPP in scientific research; and the availability of large machine configurations during development (cf., the management policy of the Concurrent Supercomputing Consortium).

Furthermore, the present peer review of such centers must be modified to enable a 'cycle-shop' and 'development center' to coexist. To merely judge such centers on the basis of CPU cycles delivered is not sympathetic to the generation of new application codes. There is a growing need for the support offered by such centers to be more application-area focused - to provide in-house expertise, a repository for algorithms, and a focal point for the communication between computational chemists, computational scientists, computer scientists, and hardware vendors. 


\subsection{Summary and Conclusions}

In this section we summarize and draw conclusions based on the material presented above. We first focus on those points relevant to application development and then consider the impediments to such developments. It is quite clear to this panel that both algorithmic advances and removal of some of the impediments described above are essential in meeting the challenges faced by the computational chemistry community. The set of eight specific recommendations presented as part of the Executive Summary are based on this analysis and on this panel's expertise in high-performance computational chemistry. It is the panel's belief that establishing the priorities of these recommendations is best left to the community and appropriate funding agencies.

\subsection{State-of-the-Art Software Developments}

Some unattractive characteristics of the early parallel machines included slow floating point performance, poor $\mathrm{I} / \mathrm{O}$ performance, small memories (per node), and significant operating system restrictions (e.g., lack of multitasking on each node). These features, along with a lack of standardization, have also contributed to the disturbingly slow progress in the development of scalable, low-level applications libraries (e.g., linear algebra libraries and, in particular, scalable and efficient routines for the ubiquitous matrix eigenvalue problem). More recent machines are addressing these hardware shortcomings. The use of inexpensive RISC CPUs addresses not only the issue of floating point performance, but allows some degree of portability between desktop machines, small-scale parallel machines, and MPP machines. Improved communications, coupled sometimes with increasingly inexpensive local disk space, are beginning to address the $\mathrm{I} / \mathrm{O}$ performance issues. Local memories of MPPs are often comparable in size to those of workstation memories and, when coupled with the trend toward more standard operating systems (or kernels), extend the range of applicable algorithms and facilitates code and algorithm development. Specific points noted by the panel include the following:

- The current trend to workstation clustering, and the improved availability of parallel computers and portable programming tools, are resulting in many groups making serious attempts to make practical use of this technology. Indeed, significant progress has been made in the use of small clusters of distributed workstations. Computational chemistry calculations with minimal communications requirements can be run very efficiently (and cost effectively) on clusters of workstations connected with standard networks (e.g., ethernet and FDDI). These can easily support 8 to 16 machines running as a dedicated parallel cluster and deliver vector supercomputer performance. Such a hardware arrangement provides a viable platform for production science as well as algorithm testing and development.

- While an increasing variety of parallel languages, toolkits, and new architectures are, or soon will, be available, these neither solve all portability problems nor make it obvious which parallel strategies should be adopted.

- We cannot emphasize sufficiently the importance of performance modeling. Most performance problems (e.g., sequential bottlenecks, I/O bottlenecks, load imbalance, communication contention, 
queuing) may be predicted by a simple model constructed with little or no program development. Such modeling is also essential to compare the performance and scalability of different algorithms. Central to the development of performance models is knowledge of fundamental machine characteristics (e.g., latencies and bandwidths, network topology, processor speeds, I/O rates), which are amenable to measurement.

- Poor reliability, slow $\mathrm{I} / \mathrm{O}$, small memory, limited disk space, and inadequate support continue to make production usage of current machines difficult.

- Development time is becoming increasingly available on a variety of parallel machines, but production time remains difficult to acquire.

- Better support for infrastructure issues (e.g., training and development on parallel machines) is required.

- Distributed data algorithms will be required for scalable massively parallel computations.

- More memory per node and faster and more parallel I/O will be necessary for future development of fast and scalable algorithms.

\subsubsection{Quantum Chemistry}

We began this report suggesting that parallel computers were now able to deliver on the promises of greater performance, larger calculations, and cheaper computation. Several ab initio methods now exist that satisfy all three criteria. However, it is clear that a particular application really needs only to satisfy one of these three criteria in order to justify execution on a parallel machine.

- It is evident that distributed-data algorithms are essential to exploit fully and cost effectively the performance of MPPs. Fully-distributed algorithms, in addition to being scalable, can sometimes be more CPU-efficient than replicated data algorithms because of improved load balancing and overlapping of computation and communication. However, we emphasize scalability and balanced usage of all machine resources, rather than just CPU-efficiency. It seems that there are now available three scalable direct-SCF algorithms (Colvin, Furlani, and Harrison) of differing efficiency and complexity.

- Recent developments exploit various numerical representations and approximations to substantially decrease the cost of Fock matrix construction. No parallel implementations of these approaches have yet been reported in the literature.

- Progress with DFT, CI and many-body methods is also very promising. Fundamental to most of these advances is the tailoring of algorithms to accommodate NUMA, and the development of the global array tools is motivated by this observation.

- Replicated-data algorithms will continue to be used and developed. The modestly parallel workstation clusters that are so widely available are ideally suited to the execution of large problems (e.g., an 800 basis function, no symmetry, SCF geometry optimization) with replicated 
data algorithms. For such coarse-grained parallel calculations, the reduced time-to-solution often compensates the minimal loss of parallel efficiency. Much larger calculations, or larger clusters, will again demand distributed algorithms.

\subsubsection{Reactive Scattering}

- Fully-scalable codes in some applications of reactive scattering for MPPs are already becoming available and a rapid proliferation of such codes for different methods is expected.

- Although some codes could make use of Teraflop machines today by exploiting loosely coupled parallelism, the majority of codes are not scalable. (We may need to have the machines to learn how to use them.)

- Tight coupling between quantum dynamics, electronic structure, and experiment for systems having a small number of atoms can, for the first time, become possible with large MPP machines, opening up a new era in chemical dynamics.

- Concurrent support for high-accuracy electronic structure parallel code development for few atom systems is recommended.

- The availability of large MPP machines for such work is recommended.

\subsubsection{Molecular Dynamics}

- Notwithstanding the algorithmic developments described in Section 2.1, and the laudable efforts by the simulation community to exploit fully available parallel hardware, the problem remains, in macromolecular structure at least, that the current time and spatial scales are, in many instances, inappropriate for the target physical questions. Given that information spanning several orders of magnitude beyond the currently accessible picosecond-nanosecond time frame is needed from MD simulations, the development of more effective multiple-timescale MD methods is seen as crucial.

- When assessing the algorithmic developments required to achieve the computational goals of MD simulations, there are two quite distinct requirements. 1) The development of algorithms, which are effective for a modest number of atoms (typically $N<1000$ ). The aim in any simulation must be to model, to a sufficient level of accuracy, the required physical effects with the smallest possible value of $\mathrm{N}$. The vast majority of MD simulations continue to be carried out on systems with between several hundred and a few thousand atoms. 2) In the area of large MD simulations, the development of truly scalable algorithms capable of exploiting larger and faster parallel machines. Algorithms that scale optimally with both the number of atoms and the number of processors will leave the simulation community ideally positioned to tackle larger problems as the next generation of more powerful parallel machines become available.

- A comparison of the results of a standard Lennard Jones (LJ ) benchmark problem (using atom-, force-, and spatial-decomposition algorithms) on three parallel supercomputers with CRAY Y-MP and C90 timings reveals the current generation of parallel machines to be competitive with conventional vector supercomputers, even for small problems. For large problems, the spatial 
algorithm achieved parallel efficiencies of $90 \%$, with the Intel Delta performing about 30 times faster than a single Y-MP processor and 12 times faster than a single C90 processor. Atomdecomposition methods are the most straightforward to implement, reflected by their usage in major simulation packages such as CHARMM and GROMOS. Yet their dependence on global communications limits the number of processors that can be effectively used. Force-decomposition algorithms based on a block-decomposition of the force matrix reduces the memory and communication costs by a factor of _ $P$, versus the atom-decomposition algorithms. Spatialdecomposition algorithms are well known to be ideally suited for very large MD simulations. In the large $N$ limit, optimal $O(N / P)$ scaling should be possible, making it clearly the fastest algorithm, assuming that good load-balance is also achievable. An efficient implementation is, however, more complex than that encountered with either the atom- or force-decomposition methods, so that integration into an existing serial code would involve a substantial reworking of data structures and code.

- Considering long-range $\mathrm{MD}$, a variety of approximate methods have been developed to overcome the $\mathrm{N}^{2}$ bottleneck that characterizes the forces treatment, including particle-mesh algorithms, hierarchical methods, and fast-multipole methods. One of the most promising developments is the Cell Multipole Method (CMM) which, scaling linearly with $\mathrm{N}$, requires only modest memory and is well-suited to highly parallel and vector computers

- While simulation packages (CHARMM, DISCOVER, GROMOS, and AMBER) have probably made the largest strides in exploiting the potential of parallelism, the scalability of present parallel implementations is limited to, at best, a few dozen processors.

- The widespread adoption by the simulation community of a specific benchmark problem to gauge both the effectiveness and scalability of emerging algorithms, and the suitability of different machine architectures, is to be applauded.

- There can be little doubt, based on the wealth of experience to date, that the MIMD programming model is the only one that provides sufficient flexibility to embrace all the data structures and computational enhancements that are routinely exploited in MD codes on serial and vector machines.

- Synergism between combined developments in quantum chemistry and molecular dynamics for treatment of chemical reactions in condensed and biophysical system will lead to new insight and should be strongly supported.

- There is a critical need for data analysis "on the fly", where the simulation is so large that the data cannot efficiently or practically be stored during the simulation. 


\subsection{Impediments to the Migration, Development, and Exploitation of Computational Chemistry Software}

\subsubsection{Education and Training}

The panel recommends a continued and expanded effort in computational chemistry methods development in both academia and the national laboratory community, and a strengthened integration of computer science and computational chemistry training, through strong interdisciplinary undergraduate and graduate programs that emphasize the methods, algorithms, tools, and architectures required in large-scale scientific computations.

\subsubsection{An Integrated Massively Parallel Processor and Distributed Computing Environment for Chemistry}

Computational chemistry represents a diverse set of algorithms that have little in common with the data-parallel algorithms of other disciplines that have been better represented to programming tool and hardware designers. There is a need for software tools (programming, operating system support, compilers, etc.) that allow computational chemists to port, develop, and analyze both existing and new applications. An improved relationship between methods developers and those designing machines, targeted to encourage the incorporation of features that better support chemistry algorithms, should be coordinated by the community and supported by the various funding agencies.

\subsubsection{The Integration of Computational Communities}

The computational chemistry community needs to establish a collective interaction with computer vendors, the computer scientists, and their industrial counterparts. This interaction should be encouraged by the establishment of workshops that develop and integrate computational chemistry computer architecture and software requirements. The computational chemistry community must encourage vendor, computer scientist, and industrial participation in these workshops, looking to both accelerate and, subsequently, support the transfer of MPP technology to industry.

\subsubsection{Computational Chemistry Benchmarks}

A suite of benchmarks should be established that truly reflects the demands of chemistry applications (cf., the NAS kernels in CFD) and that will act to raise the profile of computational chemistry. These should be drawn from those areas that are identified as driving high-performance computing and should address and highlight the current problems and potential of MPP.

\subsubsection{Configuration and Operation of Massively Parallel Processor Centers}

Successful exploitation of present HPCC-MPP centers by the computational chemistry community requires an associated support infrastructure and management that is more responsive to this community. While the hardware at the center should be configured to truly reflect the demands of the community (disk storage, memory, networking, etc.), center management should be more receptive to the need for both production and development time (with its associated low CPU utilization). Centers should act as a repository for algorithms and expertise, and act as a focal point for communication between chemists and computers scientists, vendors, numerical experts, etc. 



\section{Distribution}

No. of

Copies

\section{OFFSITE}

2 DOE/Office of Scientific and Technical Information

\section{J. Almlöf}

Department of Chemistry

University of Minnesota

Minneapolis, MN 55455

D. Boyd

Department of Chemistry

Indiana University-Purdue University

at Indianapolis

402 North Blackford Street

Indianapolis, IN 46202

M. Broido

Acting Program Manager, EMSL

Project

Office of Health and Environmental

Research

U.S. Department of Energy

19901 Germantown Road, ER-74

Germantown, MD 20874

B. R. Brooks

National Institute of Health

9000 Rockville Pike

Bethesda, MD 20892

C. L. Brooks III

Department of Chemistry

Carnegie Mellon University

4400 Fifth Avenue

Pittsburgh, PA 15213
No. of

Copies

J. S. Cavallini

ER-30

GTN

U.S. Department of Energy

Washington, DC 20585

M. Colvin

Center of Computational Engineering

Sandia National Laboratory

Livermore, CA 94551

D. A'. Dixon

Du Pont Research and Development

Route 141 Murphy Road

Experimental Station

Wilmington, DE 19880-0328

M. Dupuis

IBM Corp.

Dept. 48B/MS 428

Neighborhood Road

Kingston, NY 12401

S. T. Elbert

Ames Laboratory/USDOE

315A Harley Wilhelm Hall

Ames, IA 50011-3020

J. T. Golab

Chemistry Modeling Group

MC C-6

150 West Warrenville Road

Amoco Research Center

Naperville, IL 60563-8460

Distr.1 
No. of

\section{Copies}

M. S. Gordon

Department of Chemistry

Iowa State University

Ames, IA 50011

M. F. Guest

SERC Daresbury Laboratory

Warrington WA4 4AD

ENGLAND

R. Hilderbrandt

Room 1055S

National Science Foundation

4201 Wilson Boulevard

Arlington, VA 22230

M. Kalos

Cornell Theory Center

Cornell University

Ithaca, NY 14853

W. H. Kirchhoff

ER-141

GTN

U.S. Department of Energy

Washington, DC 20585

T. A. Kitchens

Office of Scientific Computing

ER-30

GTN, G-236

Washington, DC 20545

A. Kuppermann

Department of Chemistry

California Institute of Technology

1201 East California Boulevard

Pasadena, CA 91125

A. H. Laufer

ER-141

GTN

U.S. Department of Energy

Washington, DC 20585
No. of

Copies

K. B. Lipkowitz

Department of Chemistry

Indiana University-Purdue University at Indianapolis

402 North Blackford Street

Indianapolis, IN 46202

R. S. Marianelli

ER-14

GTN

U.S. Department of Energy

Washington, DC 20585

V. McCoy

Department of Chemistry

California Institute of Technology

1201 East California Boulevard

Pasadena, CA 91125

B. McCurdy

National Energy Research

Supercomputer Center

Lawrence Livermore National

Laboratory

Livermore, CA 94551

P. Messina

CCSF

California Institute of Technology

1201 East California Boulevard

Pasadena, CA 91125

D. Nelson

ER-30

FORS

U.S. Department of Energy

Washington, DC 20585

W. P. Reinhardt

Department of Chemistry

BG-10

University of Washington

Seattle, WA 98195 
No. of

Copies

A. P. Rendell

SERC Daresbury Laboratory

Warrington WA4 4AD

ENGLAND

J. Saltz

Department of Computer Science

University of Maryland

College Park, MD 20742

K. Schulten

Department of Chemistry

University of Illinois

505 South Mathews Avenue

Urbana, IL 61801

R. Stevens

Math and Computer Science Division

Argonne National Laboratory

Argonne, IL 60439

P. Taylor

San Diego Supercomputer Center

University of California at San Diego

PO Box 85608

San Diego, CA 92138
No. of

Copies

A. F. Wagner

Theoretical Chemistry Group

Chemistry Division

Argonne National Laboratory

Argonne, IL 60439

\section{ONSITE}

2 DOE Richland Operations Office

H. E. Bell

K8-50

J. P. Neath

K8-50

33 Pacific Northwest Laboratory

R. A. Bair K1-87

C. A. Counts K2-20

T. H. Dunning, Jr. K2-20

R. A. Eades K1-83

B. C. Garrett K1-83

R. J. Harrison K1-90

J. A. Nichols K1-96

R. A. Kendall (20) K1-90

R. J. Littlefield K7-15

Technical Report Files (5) 